Elsevier required licence: (C) $<2020>$. This manuscript version is made available under the CC-BY-NCND 4.0 license http://creativecommons.org/licenses/by-nc-nd/4.0/

The definitive publisher version is available online at

[https://www.sciencedirect.com/science/article/pii/S016740481930197X?via\%3Dihub] 


\title{
PrivySharing: A Blockchain-Based Framework for Privacy-Preserving and Secure Data Sharing in Smart Cities
}

\author{
Imran Makhdoom ${ }^{1}$, Ian Zhou ${ }^{2}$, Mehran Abolhasan $^{3}$, Justin Lipman $^{4}$, Wei Ni ${ }^{5}$ \\ 1,2,3 University of Technology, Sydney, Australia \\ ${ }^{4}$ Food Agility CRC Ltd, University of Technology Sydney, Australia, ${ }^{5}$ Data61-CSIRO \\ imran.makhdoom@uts.edu.au ${ }^{1}$, ian.zhou@student.uts.edu.au ${ }^{2}$ mehran.abolhasan@uts.edu.au ${ }^{3}, J u s t i n . L i p m a n @ u t s . e d u . a u^{4}$, \\ Wei.Ni@data61.csiro.au
}

\begin{abstract}
The ubiquitous use of Internet of Things (IoT) ranges from industrial control systems to e-Health, e-commerce, smart cities, agriculture, supply chain management, smart cars, cyber-physical systems and a lot more. However, the data collected and processed by IoT systems especially the ones with centralized control are vulnerable to availability, integrity, and privacy threats. Hence, we present "PrivySharing," a blockchainbased innovative framework for privacy-preserving and secure IoT data sharing in a smart city environment. The proposed scheme is distinct from existing strategies on many aspects. The data privacy is preserved by dividing the blockchain network into various channels, where every channel comprises a finite number of authorized organizations and processes a specific type of data such as health, smart car, smart energy or financial details. Moreover, access to users' data within a channel is controlled by embedding access control rules in the smart contracts. In addition, data within a channel is further isolated and secured by using private data collection and encryption respectively. Likewise, the REST API that enables clients to interact with the blockchain network has dual security in the form of an API Key and OAuth 2.0. The proposed solution conforms to some of the significant requirements outlined in the European Union General Data Protection Regulation. We also present a system of reward in the form of a digital token named "PrivyCoin" for users sharing their data with stakeholders/third parties. Lastly, the experimental outcomes advocate that a multi-channel blockchain scales well as compared to a single-channel blockchain system.
\end{abstract}

Keywords: Internet of Things, Smart City, Security and Privacy, Blockchain, EU GDPR Compliance.

\section{Introduction}

There has been an exponential growth in the IoT-based services in the world, especially in telehealth, manufacturing and in urban areas to form smart cities. IoT is expected to connect 30 billion devices by 2020 1. Use of IoT technologies will not only improve the quality of life of people but also contribute to

5 the world economy. IoT is predicted to create about USD 7.1 trillion contributions to the global economy by 2020 [1. Concurrently, it is also estimated that by 2030 the global urban population will reach 5 billion. This rapid urbanization demands effective, and optimum use of city resources as well as smart governance and efficient service delivery [2, 3. It is believed that the solution to the rapid urbanization problems lies in creating a smart city that utilizes IoT technologies to monitor the physical world in real-time and provide 10 intelligent services. These services may include eToll, smart parking $\underline{3}$, smart health (remote patient monitoring, health emergency response), and police assistance (for law and order situations, e.g., riots, crime, or security breaches) 2]. However, at the same time, IoT devices are vulnerable to a vast number of security and privacy attacks [4. Although, these threats are known to the manufacturers, unfortunately security in IoT devices is either neglected (due to cost or lack of expertise) or treated as an afterthought [5]. Similarly,

15 a smart city network also suffers from numerous security and privacy issues [2, 6], such as threats to privacy, integrity, and availability of user data, false data injection [3], vulnerability to Sybil Attack [7, and single point of failure due to centralized control. 


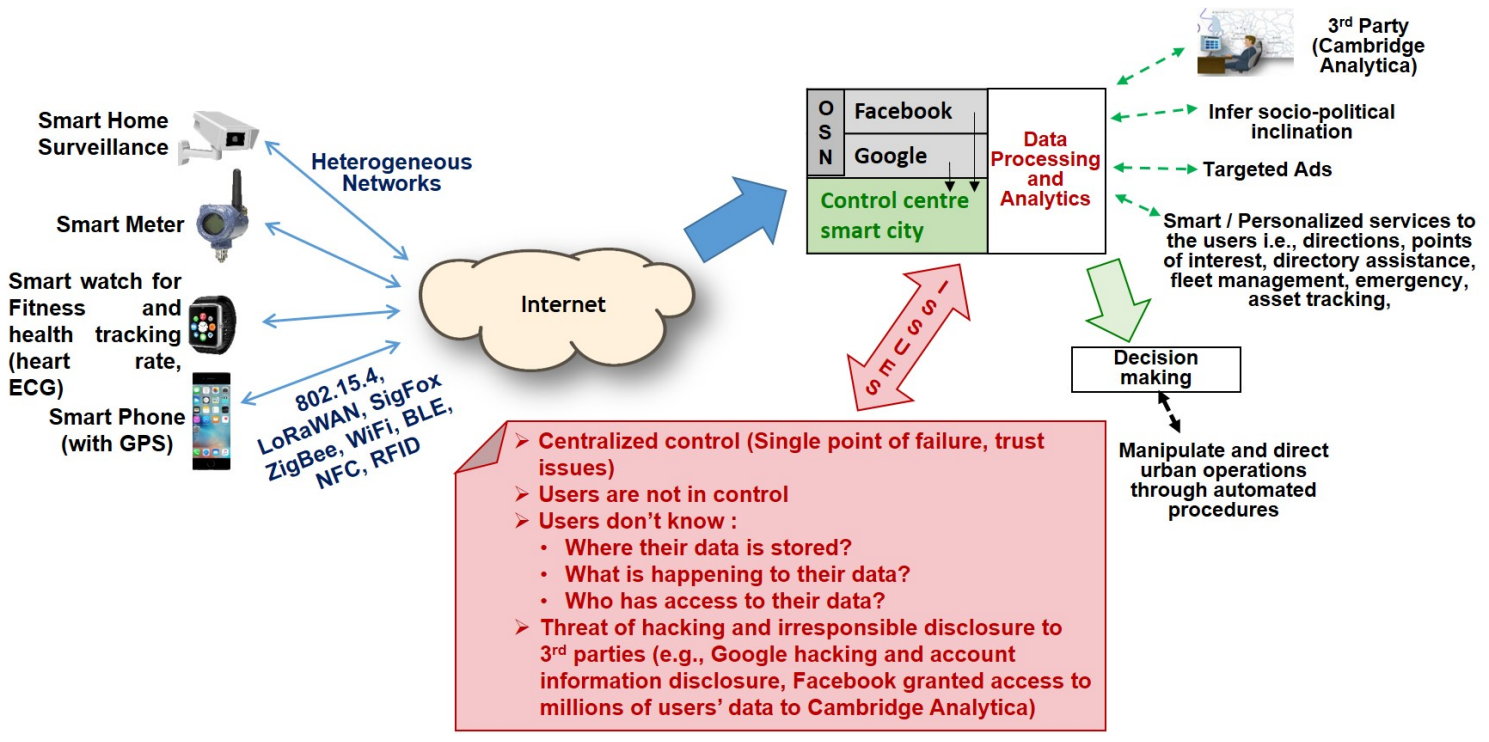

Figure 1: Issues in the smart city environment

If we look at Figure-1, the user data collected by numerous sensors is stored and processed by various OSN (Online Social Networks), smart city control center or various other smart city components such as Intelligent Transportation Systems (ITS), health emergency response, fire and rescue, etc., These components (with mostly centralized control) process user data for the provision of various services to the users and third parties. Although such a centralized control may look effective from the outside, yet it has some significant security concerns.

Centralized control is subject to a single point of failure in case of a cyber-attack or other technical 25 malfunctions [8. Moreover, it also has trust issues, as the users have to put their trust in the entity that is handling their data. Hence, users have no control over their data assets. Further concerns for user data include: Users do not know where their data is stored and what is happening to it. Who has access to it, and is there any unauthorized disclosure to the third parties. The above-mentioned users' concerns are very much real as the disclosure of personal data leakage concerning millions of users by Facebook Inc. 19, 10, and 30 a bug in Google Plus [1] that resulted in the exposure of personal information of approx 500,000 users is a candid example of one of cloud/OSN vulnerabilities.

Moreover, any smart city application is believed to store, process and analyze users' data. Hence, every security solution developed for a smart city environment must comply with the undermentioned key requirements of European Union General Data Protection Regulation (EU GDPR) [12] while handling users' 35 data:

- Personal data should be processed only with the consent of the data owner.

- Any technology dependent on user data must preserve user privacy by design.

- The gathering, processing or use of personal data should be in accordance with the instructions based on a mutual contract between the user and the third parties.

- The owner of data has the right to access the information concerning the processing of his data, i.e., which third parties have access to what data and how they use it.

- It is the right of the data owners that their data be erased immediately once it is no longer needed.

- The system should be transparent such that individuals know about the collection and use of their data. 
${ }_{45}$ As far as IoT security is concerned, researchers and security analysts are trying to leverage cryptographic security benefits of blockchain to resolve security and privacy issues of IoT. Hence, we believe that a carefully selected blockchain technology with an insightful business network design can resolve most of the data integrity and privacy issues of a smart city.

\subsection{Related Work}

Security researchers around the world are developing and investigating ingenious ways to implement blockchain in the IoT environment. These use cases aim to take advantage of the inherent benefits of the blockchain such as decentralized control, immutability, cryptographic security, fault tolerance, and capability to run smart contracts. Recently, researchers 13 presented a blockchain-based data sharing framework for a smart city environment. The framework called "SpeedyChain" focuses on reducing the TX settlement time

55 for real-time applications such as smart cars and also aims to ensure user privacy. Moreover, it ensures data integrity, tamper-resistance, and non-repudiation that are some of the intrinsic benefits of the blockchain. In another work, Pradip Kumar and Jong Hyuk proposed a Software Defined Networking (SDN) and blockchainbased hybrid network architecture for a smart city [14. The proposed architecture addresses usual smart city issues including high TX latency, security and privacy, bandwidth bottlenecks, and requirement of high

${ }_{60}$ computational resources. In the proposed model the smart city network is divided into a distributed core network comprising resourceful miner nodes and the centralized edge network constituting inept devices. The edge nodes store access policies for locally registered nodes. Authors claim that in addition to reducing TX latency, and reduced network bandwidth, the proposed model limits the effects of a node compromise to the local area.

65 Additionally, authors in 15 proposed a smart contract based sharing economy services in a smart city. The proposed model uses Artificial Intelligence (AI) for data analytics and also uses blockchain to store the results. Similarly, Biswas and Muthukkumarasamy [16] presented an overview of a blockchain-based security framework for secure communication between smart city entities. Authors claim that the integration of the blockchain with devices in the smart city will provide a shared platform where all the devices would be able

70 to communicate securely. However, the researchers did not disclose some necessary details about the type of blockchain platform, consensus protocol, and TX/block validation techniques adopted in the smart city application.

In another endeavor [17, 18], security researchers have proposed solutions to address various user privacy issues in ITS. Nonetheless, they do not cater to the challenges of smart cities such as trustless data 75 sharing among multiple organizations. Similarly, Ali Dorri and Raja Jurdak proposed a secure, private and lightweight architecture of a blockchain-based smart home application 19, 20. It aims to solve certain blockchain issues such as computational intensiveness, latency in TX confirmation and energy consumption. To reduce computational overhead, and energy consumption each block is mined without any Proof of Work (PoW). Moreover, the latency in TX confirmation is reduced by considering a TX true, whether it is mined

${ }_{30}$ in a block or not. In addition, the proposed scheme utilizes cloud storage to ease up the memory requirements for smart home devices. However, there are many security concerns that need further explanation with logical reasoning [21]. Likewise, another team of researchers proposed an Ethereum Blockchain [22] based mechanism to manage IoT devices [23]. Nonetheless, Ethereum Blockchain does not provide data privacy.

In another work, to avoid issues concerning the single point of failure in a centralized system, researchers

85 proposed an Ethereum Blockchain based decentralized, self-managing Vehicular Ad-Hoc Network (VANET) with a challenge-response based authentication [24. However, the proposed scheme does not explain the procedure of consensus and block mining. There is also no discussion about the type of information to be published on the blockchain and the latency in TX confirmation. Above all, Ethereum Blockchain does not provide data privacy and confidentiality.

Correspondingly, Yu Zhang and Jiangtao Wen proposed an Ethereum Blockchain based decentralized electronic business model for the IoT 25 . However, the proposed solution mostly focused on the working of the e-business model, so there is a lack of discussion on technical aspects such as block mining mechanism, modalities of implementing blockchain on IoT devices, and the methodology of achieving data confidentiality and privacy. Similarly, in another work [26] authors introduced a blockchain-based security framework for

95 IoT implementations. Nonetheless, the proposed solution focuses on data authentication and secure communication between the sensor devices and the controllers. The researchers make use of the received signal 
strength (RSSI) of the message sent by a sensor device as a parameter to ensure the randomness of data to avoid replay and data forging attacks by a MITM (Man-in-the-Middle) attacker. Few other researchers have also proposed a blockchain-based approach of exchanging data in the smart city between nontrusted 100 organizations 27. In this regard, if a third party queries some data, e.g. a credit report concerning a user, then the executor node gets the input in the form of private data from the respective organization through a local private API. The data is encrypted with an organization's private key and is decrypted once in the executor sandbox using the organization's public key. Hence, the querying party receives only the processed data and does not see the original data itself.

Since the GDPR legislation came in to effect on $25^{\text {th }}$ May 2018, researchers have been working on various aspects of data protection to develop GDPR compliant data protection/processing frameworks. In this endeavor, 28 proposed a blockchain-based design concept for developing GDPR compliant data management platforms. The solicited framework shares, and revoke the sharing of user data only with the consent of data owner. Moreover, the blockchain-based framework can also endorse the service providers for being correctly ${ }_{10}$ following the GDPR policies or not. As per the devised concept, only data owners and data controllers can create, update, and withdraw consent, and only authorized entities can process user data. The proposed mechanism uses blockchain to handle authentication, authorization, and data access control token validation. Whereas, the data is stored in a centralized resource server that is assumed to be a trusted party. Apart from the resource server being a trusted party, the proposed solution does provide some security guarantees; however, it seems to have high communication complexity. As authors also claim that due to increased message overhead, the proposed scheme does not support high performance and scalability since the TX latency significantly increases and throughput decreases with the increase in the number of nodes. Similarly, [29] recommended a conceptual architecture of a human-centric and GDPR compliant blockchain-based personal data and identity management system (BPDIMS). The authors focus on designing a framework, which is 20 transparent and provides data owners the full control over the usage of their data. The researchers address specific issues concerning data usage, i.e., user consent, transparency of data processing, purging of user consent, reward mechanism for users, data integrity, and confidentiality. However, this work is still at conceptual stages and does not present any technical details or performance evaluation.

Similarly, Ricardo et al. proffered a blockchain-based scheme to facilitate data accountability and provenance tracking [30. Data provenance tracking is achieved by maintaining a list of references to the data provided to the controller. The list is updated whenever some data is sent to the data controller/service provider. Whereas, data accountability is accomplished by specifying restrictions on data usage in smart contracts. The restrictions are defined under the domain of a preventive mechanism, using a security policy language recommended by SecKit (Model-based Security Toolkit). The preventive mechanism denies actions 30 such as allow, deny, modify, or delay the operations concerning data usage to the data controllers. The authors primarily discuss various design choices for the data usage contract models while considering the provision of maximum data provenance information to the data owners in a trusted and privacy friendlymanner. The sample contract models are evaluated based on gas consumption in Ethereum Virtual Machine (EVM). Correspondingly, authors in 31 introduced a consent management platform named ADvoCATE ${ }_{35}$ for IoT data processing. ADvoCATE uses Ethereum Blockchain to preserve the integrity of users' consents and related updated versions. The ADvoCATE may be interpreted as a cloud service platform with various components such as blockchain, intelligent policy analyzer, consent notary, and storage. The consents notary ensures that the created consents are up to date and are also protected against unauthorized modification. Whereas, the intelligence component makes use of Fuzzy Cognitive Maps (FCM) methodology to identify any rules/policies that contradict with GDPR requirements concerning the handling of users' data. Moreover, whenever an IoT device is installed, the user gives his consent to the data controller/service provider through a smart contract to access IoT device data. The digital consents duly signed by the data controller and the device owner are stored on the ADvoCATE platform, whereas, the blockchain stores only the hashes of these consents for integrity. However, the proposed platform is still in the development phase and has not been 145 extensively tested or evaluated. The authors only highlight the gas (ether) consumption of smart contracts, and there is no analysis on TX latency, TX throughput, scalability, or communications overhead.

In a similar endeavor 32, Nesrine Kaaniche and Maryline Laurent, presented a blockchain-based data usage auditing architecture that provides the data controllers with unforgeable evidence of users' consent. The researchers claim to provide user anonymity by letting the data owners (which are delegated PKG) create 
150 a distinctive public-private key pair for each smart contract they initiate to share data with a service provider or a data processor. Moreover, the authors used hierarchical ID-based encryption to prevent unauthorized disclosure. The data is stored on off-blockchain storage, whereas blockchain smart contracts are used to store the hash of data and data usage policy. Also, there is a specific smart contract between the data owner and every other service provider or data processor. However, the architecture is not supported by any performance evaluation, e.g., TX settlement time, block commit time, or latency. In another work, authors evaluated the potential use of blockchain technology to facilitate the transformation of institution-centric exchange of data to patient-centric, and patient-driven data sharing [33. The researchers recommend that the blockchain can be used to provide transparency over the state of shared data, and related TXs among different stakeholders. In that permissioned blockchains can be more productive in terms of delivering strict access control concern160 ing read-write permissions over users' health data. Authors also believe that the blockchain provides a lower cost of TX verification and data integrity as compared to the traditional systems. It is also accredited that the blockchain can also ensure the availability, swift access, and immutability of health data. Moreover, it can also provide unique identities to all patients. However, authors foresee inevitable glitches in the use of blockchain such as high TX volume of health records, and related massive storage requirements, security, and 65 privacy issues concerning user data.

Though the research work discussed above has undoubtedly made some significant contributions towards blockchain and IoT domain. Nevertheless, there are many open issues such as preserving data privacy in a smart city environment, user-defined fine-grained access control, fast TX settlement, users' right to forget (concerning data deletion), an incentive for users to share their data, and distributed storage of user 170 data without centralized control. Therefore, to fill the respective research gaps, we propose "PrivySharing," a blockchain-based secure and privacy-preserving data sharing framework. The proposed solution aims to protect a smart city environment against most of the data integrity and privacy threats. The experimental results have shown that a carefully designed blockchain solution can ensure user data privacy and integrity in various network settings as per the wishes of the data owner. It also effectively protects against false 175 data injection and Sybil Attacks. Moreover, PrivySharing complies with some of the significant data security and privacy requirements of the European Union General Data Protection Regulation (EU GDPR). The significant contributions of this paper are:

1. Provides protection against most of the external as well as insider attacks threatening user data integrity and privacy in a smart city setting.

2. Compliance with some of the essential requirements of EU GDPR.

3. A blockchain-based solution providing the "right to forget" concerning user data.

4. A scalable (concerning blockchain size), secure, and an efficient (in terms of energy consumption and computational requirements) data sharing framework.

5. User-defined fine-grained access control to user data.

6. Providing a transparent and auditable network operation and simultaneously controlling the exposure of users' private data.

7. Secure client access to the blockchain network through a REST API.

8. A reward system for the users for sharing their data with the stakeholders/third parties.

\subsection{Basic Terminologies}

Before getting involved with the detailed architecture of PrivySharing, it is imperative to understand some terminologies specific to Hyperledger-Fabric:

- Smart Contract (SC). A SC is a sort of a digital contract based on certain rules between different organizations in the form of an executable code [34. Blockchain network uses smart contracts not only to encapsulate information but also to automate certain aspects of business TXs. Applications invoke a smart contract to generate TXs that are further recorded on the ledger. 
- Chaincode. The difference between smart contracts and chaincode is that, a smart contract defines the TX logic that updates the state of a business object contained in the world state. Whereas, a chaincode can be termed as a technical container that may contain multiple related SCs for installation and instantiation. When a chaincode is deployed, all smart contracts within it are made available to the applications 34 .

- Committing Peers. Every peer node in the Hyperledger-Fabric blockchain is a committing peer. However, a Committing Peer does not have a smart contract installed. It just validates and commits a new block of TXs sent by the Ordering Service (ODS) to its copy of the ledger 35].

- Endorsing Peers. These are special committing peers with the capability to run the smart contracts. They prepare, sign and endorse the responses to the TX proposals sent by the clients, in line with the endorsement policy of the respective channel $(\mathrm{Ch})$ 35.

- Ordering Service (ODS). It is a collection of some peer nodes that arrange the new TXs in a block and then broadcast that block to all the peers of the concerned Ch [35.

- Membership Service Provider (MSP). While Certificate Authorities (CAs) issue X.509 certificates to the network entities, an MSP states that which CAs are accepted by the blockchain network and also determine that which peer nodes are members of which organization. Different MSPs can be used to represent various organizations or multiple groups within an organization. Usually, the MSPs are defined at the network, Ch and local/peer level.

\subsection{Organization of the Paper}

\subsection{Smart City Scenario}

We assume that Alice is living in a smart city where every aspect of her life is being monitored and controlled through numerous sensors and smart devices. The critical aspects include monitoring of key health detection equipment generating security-related data and financial TXs to keep the services running. For better understanding, we have formulated a list of numerous assets (associated with a specific type of data) that Alice owns (as shown in Table-1). Based on these assets, Alice can easily decide about the permissions distinction among the stakeholders/third-parties further assists Alice to plan and control the access to her data. It is also assumed that all the registered users of the smart city network, whether offline or online, interact with each other through the PrivySharing (blockchain) APIs.

To implement the above mentioned smart city use case we have used Hyperledger-Fabric as the underlying blockchain platforms [21, 37]. Hyperledger-Fabric is a private and a permissioned blockchain that restricts 
Table 1: List of Assets

\begin{tabular}{|l|l|}
\hline Data Types & Assets \\
\hline \multirow{5}{*}{ Health Data } & - Health Alert (Heart rate, blood \\
& sugar, blood alcohol, etc.) \\
& - Full Health History \\
& - Insurance Cover \\
& - Health Payment Claims \\
& - Type of Disease \\
& - Current Disease History \\
\hline \multirow{5}{*}{ Smart Car Data } & - GPS Data \\
& - Accident Alert \\
& - Damage Assessment \\
& - Servicing and Auto Payments \\
\hline \multirow{3}{*}{ Smart Meter Data } & - Line Status \\
& - Units Consumed and Bill \\
& - Consumption Pattern \\
\hline \multirow{3}{*}{ Surveillance Data } & - Equipment Status and Servicing \\
& - Security Breach Alert \\
& - CCTV Recording \\
\hline \multirow{3}{*}{ Financial Transactions } & - Income \\
& - Expenses \\
& - Tax \\
\hline
\end{tabular}

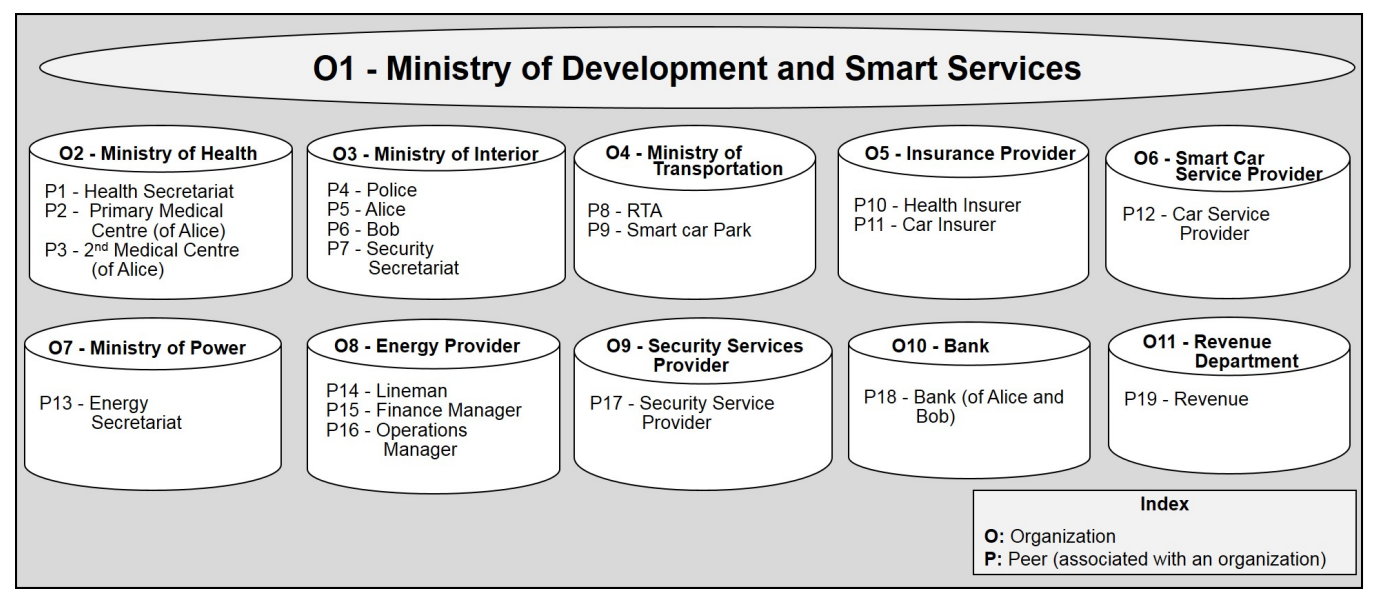

Figure 2: Network participants

participation in the network to the authorized parties only. The key feature that distinguishes HyperledgerFabric from other blockchain technologies is that in Hyperledger the blockchain ledger consists of two distinct but related parts, i.e., a blockchain to log the TXs and a world state (a database such as CouchDB [38, and LevelDB [39]) to keep track of the ledger states.

\subsection{Network Architecture}

As shown in Figure-2, we have designed a smart city blockchain network comprising eleven organizations and their associated peer nodes. Keeping in view the sharing of different categories of users' data with different stakeholders (shown in Table-2) and the requirement to ensure user data privacy and security, the blockchain network shown in Figure-3 comprises five different data Chs. Where Ch1 is used for the sharing of users' health data and organization-2 (O2), O3, and O5 are its members. Similarly, Ch2 is for smart transportation data and it comprises $\mathrm{O} 3, \mathrm{O} 4, \mathrm{O} 5$, and $\mathrm{O} 6$. Whereas $\mathrm{Ch} 3$ is for smart energy, $\mathrm{Ch} 4$ for smart security and Ch5 handles financial data (e.g., income, expenses and taxes). A Ch provides a completely separate communication mechanism between a set of organizations. Moreover, every Ch is independent of

255 the other Chs. Hence, these Chs serve to preserve the privacy of user data by securely sharing a particular type of data with authorized entities only. The network is initiated by organization-1 (O1), i.e., the Ministry 
Table 2: Assets, Stakeholders, and Access Rights

\begin{tabular}{|c|c|c|}
\hline Assets & Users / Stakeholders & Access Rights \\
\hline \multicolumn{3}{|l|}{ Health Data } \\
\hline $\begin{array}{l}\text { Health Alert (blood alcohol, } \\
\text { blood sugar, heart rate, etc.) }\end{array}$ & $\begin{array}{l}\text { - Alice } \\
\text { - Pri (Primary) Medical Center } \\
\text { - Police }\end{array}$ & $\begin{array}{l}\text { - Read } \\
\text { - Read and write } \\
\text { - Read }\end{array}$ \\
\hline Health History & $\begin{array}{l}\text { - Alice } \\
\text { - Pri Medical Center } \\
\text { - Alice and Pri Medical Center }\end{array}$ & $\begin{array}{l}\text { - Read } \\
\text { - Read } \\
\text { - Modify (Requires consent of both Alice and } \\
\text { the medical center) }\end{array}$ \\
\hline Insurance Cover & $\begin{array}{l}\text { - Alice } \\
\text { - Pri Medical Center } \\
\text { - Health Insurer } \\
\text { - Alice and Health Insurer }\end{array}$ & $\begin{array}{l}\text { - Read } \\
\text { - Read } \\
\text { - Read } \\
\text { - Modify (Requires consent of both, Alice and } \\
\text { the insurer) }\end{array}$ \\
\hline Health Payment Claims & $\begin{array}{l}\text { - Alice } \\
\text { - Pri Medical Center } \\
\text { - Health Insurer }\end{array}$ & $\begin{array}{l}\text { - Read and write } \\
\text { - Read } \\
\text { - Read }\end{array}$ \\
\hline Type of Disease & $\begin{array}{l}\text { - Alice } \\
\text { - Pri Medical Center } \\
\text { - Health Insurer } \\
\text { - Ministry of Health }\end{array}$ & $\begin{array}{l}\text { - Read } \\
\text { - Read and write } \\
\text { - Read } \\
\text { - Read }\end{array}$ \\
\hline Current Disease History & $\begin{array}{l}\text { - Alice } \\
\text { - Pri Medical Center } \\
\text { - 2nd Medical Center }\end{array}$ & $\begin{array}{l}\text { - Read } \\
\text { - Read } \\
\text { - Read }\end{array}$ \\
\hline \multicolumn{3}{|l|}{ Smart Car Data } \\
\hline GPS Data & $\begin{array}{l}\text { - Alice } \\
\text { - Car Service provider } \\
\text { - Roads/Transportation Authority (ITS) }\end{array}$ & $\begin{array}{l}\text { - Read } \\
\text { - Read } \\
\text { - Read }\end{array}$ \\
\hline Accident Alert & $\begin{array}{l}\text { - Alice } \\
\text { - Police } \\
\text { - Car Insurer }\end{array}$ & $\begin{array}{l}\text { - Read } \\
\text { - Read and write } \\
\text { - Read }\end{array}$ \\
\hline Damage Assessment & $\begin{array}{l}\text { - Alice } \\
\text { - Car Insurer } \\
\text { - Workshop }\end{array}$ & $\begin{array}{l}\text { - Read } \\
\text { - Read } \\
\text { - Read and write }\end{array}$ \\
\hline Servicing and Auto Payments & $\begin{array}{l}\text { - Alice } \\
\text { - Smart Parking } \\
\text { - Security Service Provider } \\
\text { - RTA }\end{array}$ & $\begin{array}{l}\text { - Read } \\
\text { - Read and write } \\
\text { - Read and write } \\
\text { - Read and Write }\end{array}$ \\
\hline \multicolumn{3}{|l|}{ Smart Meter Data } \\
\hline Line Status & $\begin{array}{l}\text { - Alice } \\
\text { - Lineman }\end{array}$ & $\begin{array}{l}\text { - Read } \\
\text { - Read }\end{array}$ \\
\hline Units Consumed and Bill & $\begin{array}{l}\text { - Alice } \\
\text { - Finance Manager of the Service Provider }\end{array}$ & $\begin{array}{l}\text { - Read } \\
\text { - Read and write }\end{array}$ \\
\hline Consumption Pattern & $\begin{array}{l}\text { - Alice } \\
\text { - Operations Manager of the Service Provider }\end{array}$ & $\begin{array}{l}\text { - Read } \\
\text { - Read }\end{array}$ \\
\hline Total Energy Consumption & - Ministry of Power & - Read \\
\hline \multicolumn{3}{|l|}{ Surveillance Data } \\
\hline Equipment Status and Servicing & $\begin{array}{l}\text { - Alice } \\
\text { - OEM/Service Provider }\end{array}$ & $\begin{array}{l}\text { - Read } \\
\text { - Read }\end{array}$ \\
\hline Security Breach Alert & $\begin{array}{l}\text { - Alice } \\
\text { - Police }\end{array}$ & $\begin{array}{l}\text { - Read } \\
\text { - Read }\end{array}$ \\
\hline CCTV Recording & $\begin{array}{l}\text { - Alice } \\
\text { - Police }\end{array}$ & $\begin{array}{l}\text { - Read } \\
\text { - Read }\end{array}$ \\
\hline Total Incidents of Security & - Ministry of Interior & - Read \\
\hline \multicolumn{3}{|l|}{ Financial Transactions } \\
\hline Income & $\begin{array}{l}\text { - Alice } \\
\text { - Bank } \\
\text { - Revenue }\end{array}$ & $\begin{array}{l}\text { - Read and write } \\
\text { - Read } \\
\text { - Read }\end{array}$ \\
\hline Expenses & $\begin{array}{l}\text { - Alice } \\
\text { - Bank }\end{array}$ & $\begin{array}{l}\text { - Read and write } \\
\text { - Read }\end{array}$ \\
\hline $\operatorname{Tax}$ & $\begin{array}{l}\text { - Alice } \\
\text { - Bank } \\
\text { - Revenue }\end{array}$ & $\begin{array}{l}\text { - Read } \\
\text { - Read } \\
\text { - Read and write }\end{array}$ \\
\hline
\end{tabular}




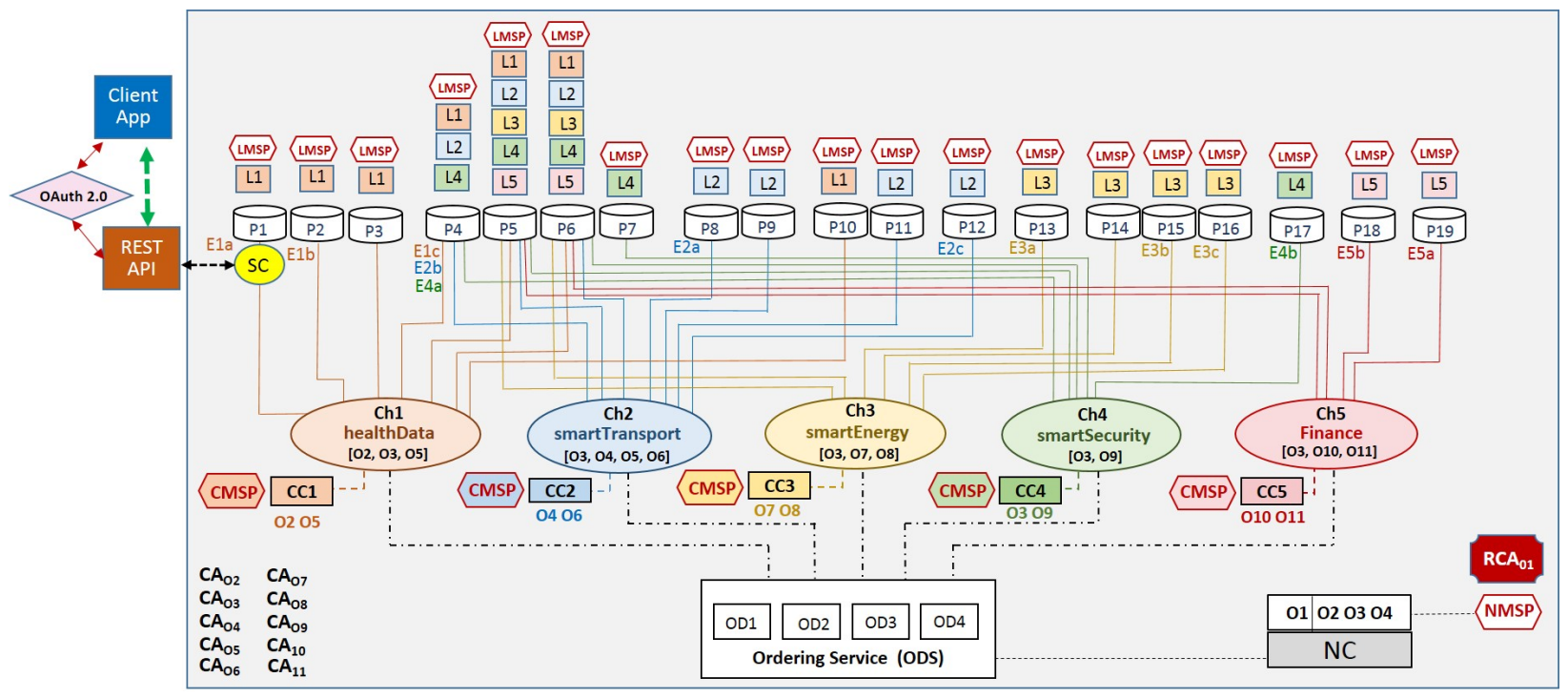

Figure 3: Smart city blockchain-network architecture

of Development and Smart Services and is governed by the policy rules specified in the network configuration (NC). NC also controls access to the smart city network. Later, O1 updates NC and gives administrative (admin) rights to $\mathrm{O} 2, \mathrm{O} 3$, and $\mathrm{O} 4$ as well. These organizations can now create consortia and Chs to add more network members. Similarly, every $\mathrm{Ch}$ is regulated by the policy rules specified in the respective Channel Configuration (CC). In this setting, Ch1 is under the control of O2 and O5 and is governed by CC1. Correspondingly, $\mathrm{Ch} 2$ is regulated by $\mathrm{CC} 2$, and so on.

The CC is essential for Ch security, e.g., if the client application (clientApp) wants to access a SC on P1, then $\mathrm{P} 1$ consults its copy of $\mathrm{CC} 1$ to determine the operations that clientApp can perform. Moreover, there 265 is a separate ledger for every $\mathrm{Ch}$, and all the peer nodes have to maintain a copy of the ledger concerning every Ch they are a member of. Therefore, if a peer, say $\mathrm{P} 4$, is a member of three different Chs, then it has to maintain three ledgers. Data in a Ch is isolated from the rest of the network including other Chs. Another important aspect of smart city blockchain network is the ODS, which is common to all the Chs. In this setup, the ODS has four ordering nodes, one each from O1, O2, O3, and O4. Each node in the ODS keeps a record of every Ch created through NC. Regarding CAs, every organization in the network can have its own CA. But there is one Root CA (RCA) in the network to establish the root of trust. As a Proof of Concept (PoC) for PrivySharing, we are using Hyperledger-Fabric RCA to issue X.509 certificates to all the network entities. These certificates serve to authenticate the network entities and to digitally sign the client application TX proposals and smart contract TX responses. A user accesses the network through a clientApp with a specific X.509 ID, using a SC. It is imperative to mention that only the endorsing peers can see the $\mathrm{SC}$ logic as they have to run the users' TX proposals to prepare the responses.

To ensure the privacy of critical user data within a Ch, i.e., keeping part of user data private from some organizations within a Ch, we adopted a methodology of "Private Data Collection," in which the critical private data is sent directly to the authorized organizations/stakeholders only. This data is stored in a private database (a.k.a sideDB) on the authorized nodes. While private information is stored on the authorized nodes, only the hash of this data is processed, i.e., endorsed, ordered, and written to the ledgers of every peer on the Ch. The hash of the data serves as evidence of the TX, and it also helps in the validation of the world state. A vital data security feature here is that the ordering nodes do not see the private data. However, to further increase the level of data privacy/confidentiality, the user has the option to encrypt his private data such that not even the peers/nodes authorized to view data stored in the private data collection can see the original contents. The data is encrypted using AES-256 bit symmetric encryption key and then stored in the private data collection. Later on, only the authorized users who have access to the decryption key can query the user's private data. Supplementary to the data encryption, there is an additional feature 


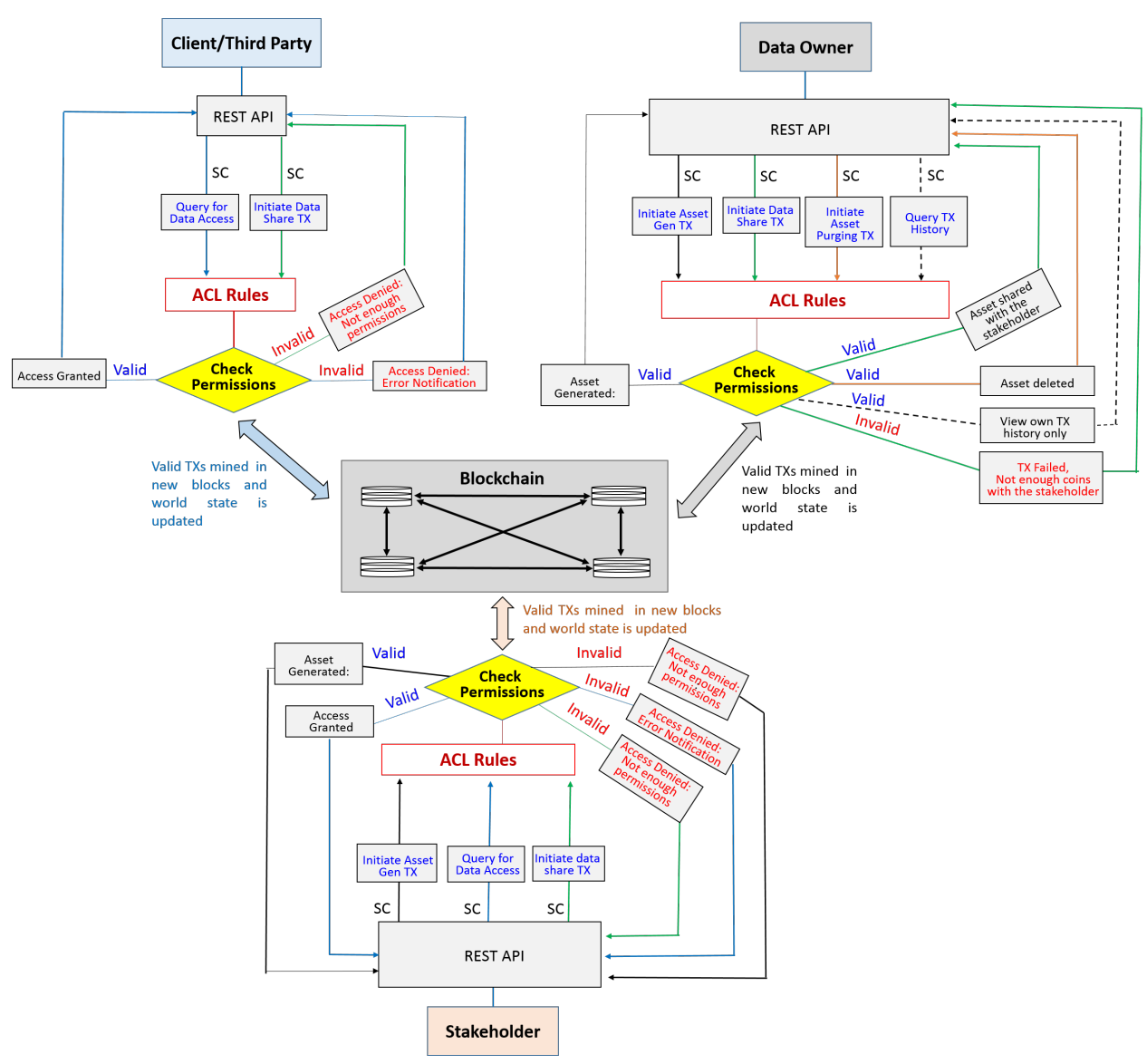

Figure 4: Smart Contract TXs

of signed encryption of private data for an increased level of user authentication and data security.

Another important feature of our proposed network architecture is the use of Membership Service Provider (MSP) at various levels such as network, Ch and local/peer. The network MSP (NMSP) defines, who all are the members of the network and who out of them have the admin rights. Additionally, an NMSP also defines that which RCAs/CAs are trusted. On the other hand, the Ch MSPs (CMSP) outline admin and participatory rights at the Ch level. All the peers and the ODS share a common CMSP to correctly authen295 ticate and verify the authorizations of the Ch members. A use case for the CMSP is that, e.g., an admin of an organization wants to instantiate a SC on Ch1, then by looking at the CMSP, the other Ch members can verify that whether that admin is a part of a specific organization or not and whether he is authorized to instantiate the SC on Ch1 or not.

Similarly, a local MSP (LMSP) is defined for every client-node/peer. The LMSP associates a peer with its organization. It also defines the permissions for that peer and allows it to authenticate itself in its TXs on the Ch. Here a question may arise that, what is the difference between CC and a CMSP. A CC contains the policies that govern that $\mathrm{Ch}$, i.e., which organizations can regulate the $\mathrm{Ch}$ and add new members. Whereas, a CMSP establishes the linkage between the nodes and their respective organizations, and what roles a node can play within a $\mathrm{Ch}$, i.e., can it instantiate a $\mathrm{SC}$ on a $\mathrm{Ch}$ ? Concerning decentralization aspect; the use of a dedicated trusted CA, a blockchain admin, and a business network admin by every organization in the blockchain network provides some degree of decentralization as compared to all the admin rights resting with a single organization.

Another question may arise that what advantages do we get by using multiple Chs for different data types as compared to a single $\mathrm{Ch}$ blockchain network to share all the types of data. There are two aspects 
310 to this selection; one is scalability, and second is increased privacy of user data. From the scalability point of view, if there is only one $\mathrm{Ch}$ for all types of data, then it means that the users will have to store the ledger comprising all those TXs that are not even related to them. Hence, the ledger size will increase rapidly, thus putting more strain on storage resources of all the users/peers. Whereas, in the case of "PrivySharing", the users will maintain a ledger that stores only that data which concerns all the users of that particular Ch. Moreover, the experimental results (Section-3) have validated that the multi-Ch blockchain network scales well as compared to a single-Ch blockchain. As far as the privacy of user data is concerned, a data specific Ch shared only by some of the stakeholders provides more privacy than a single Ch comprising all the stakeholders sharing multiple data types. Although, use of multiple data specific Chs seems scalable as compared to a single $\mathrm{Ch}$, yet the requirement for users to maintain a ledger each for every $\mathrm{Ch}$, in which they 320 participate, may still crave for ample storage resources.

PrivySharing framework has been designed, developed, and tested based on the agile blockchain application development guidelines proposed by [40]. The said guidelines helped in a systematic design, development, and testing of PrivySharing network architecture, SC functionality, and efficacy of ACL rules. Moreover, influenced by these guidelines, Figure-4 highlights different TXs initiated by various actors operating in the smart city network. Every TX and its associated decision/response based on ACL rules are depicted by the same colored line. E.g., a client/third party can only query for some user data asset. If it is authorized to access the data, the query will be successful. Otherwise, there will be an access denied error message. Both the query and respective response are shown by blue lines. Similarly, the data share TX is sketched in green color. As per PrivySharing business model the client/third party should not be allowed to submit a data sharing TX, hence, if a client still initiates a TX to share data asset of some user, then he gets a "access denied: not enough permissions" error message. TXs concerning data owner and stakeholder are also projected accordingly.

\subsection{Smart City Blockchain - Plain TX Flow}

There are two types of TXs; one is plain TX that can be viewed by all the Ch members and the other one is private data TX that is to be shared only with some selected peers in a Ch. In this regard, e.g., a plain TX that is required to update Alice's car's current location state on Ch2 is initiated by the ClientAppA installed in Alice's smart car. This TX (as shown in Figure-5 $\mathrm{a}$ ) is processed in the following steps:

Step-1. ClientAppA invokes the $\mathrm{SC}_{A}$ and sends a TX proposal containing the current location of Alice's car to the pre-defined endorsers as per $\mathrm{SC}_{A}$ endorsement policy on $\mathrm{Ch} 2$. In this case, the endorsers are $\mathrm{E} 2 \mathrm{a}$ (RTA), E2b (Police) and E2c (Car Service Provider). A TX will be approved if it is endorsed by a minimum two out of the three prescribed endorsers.

\section{Step-2}

2.1. Three endorsers E2a, E2b, and $\mathrm{E} 2 \mathrm{c}$, invoke $\mathrm{SC}_{A}$ with the proposal.

2.2. $\mathrm{SC}_{A}$ generates a query or update proposal response. The endorsers, E2a and E2b endorse the proposal

Step-3. E2a and E2b both send a signed (endorsed) TX proposal response along with RW (read, write) set back to the ClientAppA. At this stage, the endorsing peers do not apply the proposed update to their copy of the ledger.

Step-4. ClientAppA verifies that the response received from at least two endorsers is the same, i.e., de-

350 terministic. However, there is a possibility that the results were generated at different times on different peers with ledgers at different states. Hence, the peers can return different TX responses for the same TX proposal. In this case, an application can simply request a more up-to-date proposal response. Another less likely possibility is that the SC might be non-deterministic, e.g., while getting forex (foreign exchange rates) data from some websites, the TX responses can be different, as forex rates may differ at different times. Therefore, inconsistent results cannot be accepted by the application and applied to the ledger.

Step-5. Once the ClientAppA verifies the endorsers' responses, it sends the TX to the ODS.

Step-6. ODS then groups the received TXs in a block. The sequence of TXs in a block is not necessarily the same as the order of arrival of the TXs at the orderers. However, the generated blocks are final, and there are no forks. Moreover, the orderers do not host the ledger and the SCs, and they are also not concerned 360 about the value of the TX rather they just package the TXs into the blocks.

Step-7. ODS Broadcasts the next proposed block to all the peers on the Ch2. 

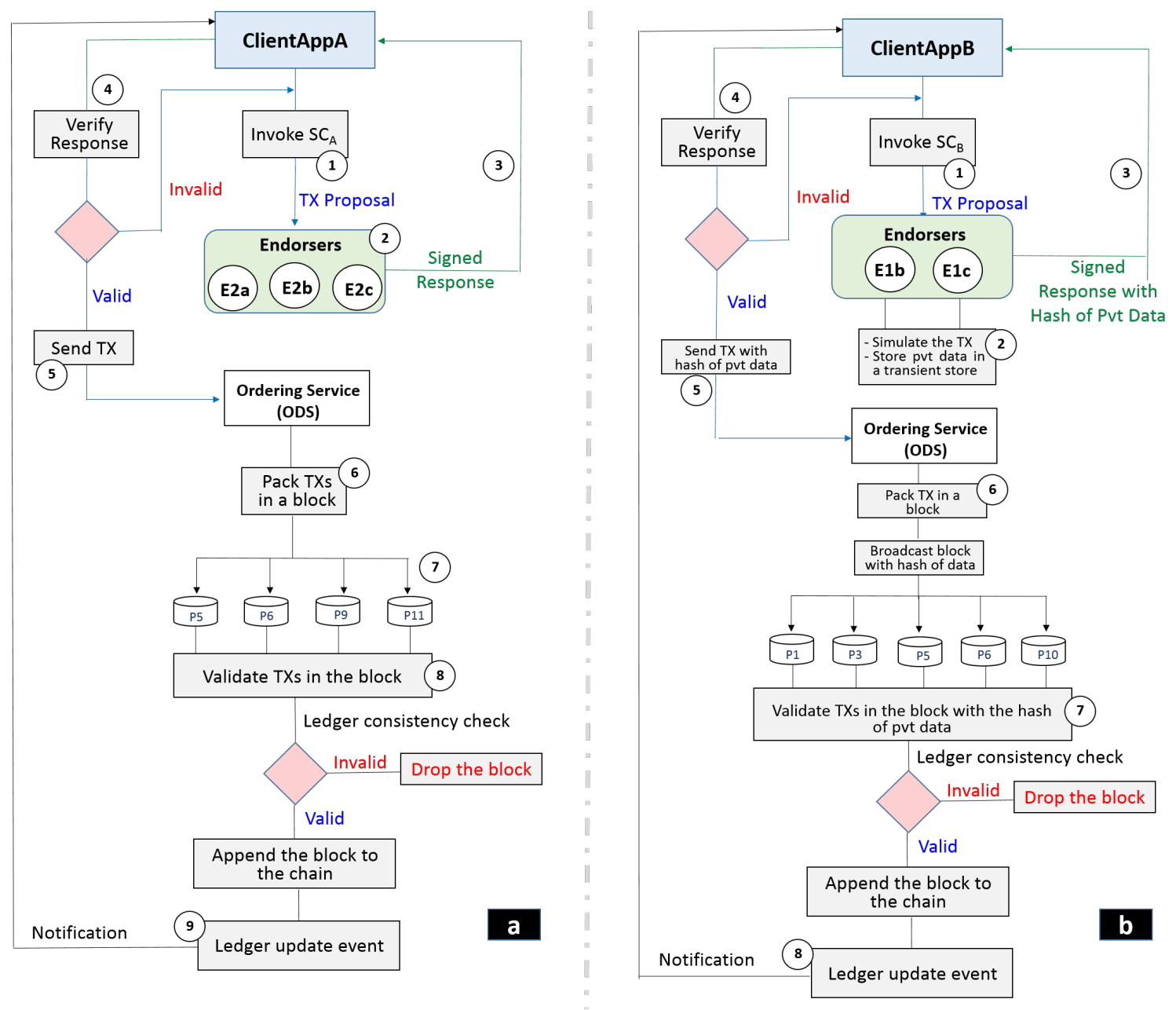

Figure 5: a) Plain TX flow, and b) Private data TX flow

Step-8. All the committing peers validate each and every TX in a block (in the same sequence as they appear in the block) to ensure that it is correctly endorsed by all the required endorsers before it is applied to the ledger. Once a TX is verified correctly, the peers perform a ledger consistency check to establish that the current state of the ledger is compatible with the state of the ledger when the proposed update was generated. World state is updated based on the validated TXs. It is to be noted that, the failed TXs are not applied to the ledger, but they are retained for audit purpose. Moreover, TX validation in Step- 8 does not require running of SCs. This is done only by the endorsers. Hence, SCs are installed only on the endorsers. This keeps the logic of the SCs confidential to the endorsing organizations only. Moreover, peers also mark ledger L2, maintained by all the peers in their file system.

Step-9. Ledger update event is generated, and the ClientAppA is notified.

It is important to note that prior to appending a block, a version check is performed to ensure that the states being updated are the same that were read during SC execution. It protects against double spending and other data integrity threats. The above mentioned TX workflow mediated by the orderers is called "Consensus", as all the peers reach on an agreement about the content and the order of the TXs. 


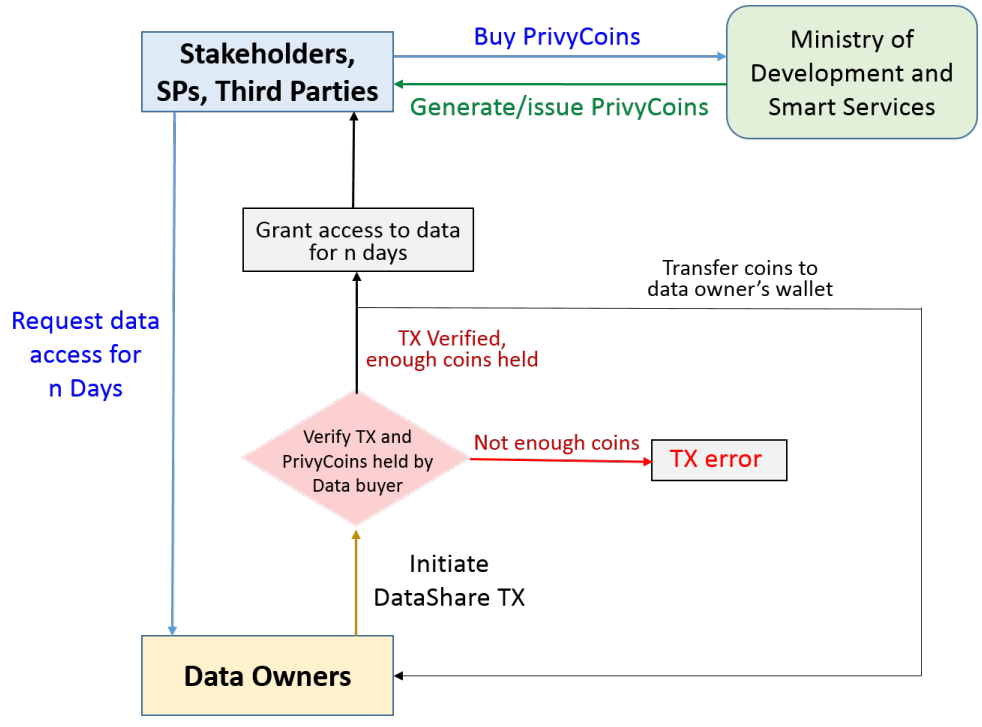

Figure 6: Reward mechanism based on PrivyCoins

\subsection{Smart City Blockchain - Private Data TX Flow}

As per smart city network settings shown in Figure-3, if a wearable blood alcohol monitoring device on Alice generates an alert to be seen only by her primary medical center and the local police for immediate response. In such a case, it is required to keep such a TX private which should not be seen by other members on Ch1 except P2, P4, and P5. Such a private data TX (as shown in Figure-5b) is processed in the following steps:

Step-1. The clientAppB submits a proposal request to invoke a SC function (RW private data) to the endorsing peers E1b (Primary Medical Center) and E1c (Police), which are part of the authorized organizations of the collection (defined by the private data dissemination policy on health alert). The private data concerning health alert on blood alcohol level is sent in a transient field of the proposal.

Step-2. E1b and E1c simulate the TX and store the private data in a transient data store (temporary storage local to them). The endorsing nodes also distribute the private data based on the collection policy to authorized peers via gossip. But in this case, we only have three peers, i.e., P2(E1b), P4(E1c) and P5.

390 Step-3. E1b and E1c send the proposal response back to the clientAppB with public data, including a hash of the private data key and value (Blood alcohol level). No private data is sent back to the clientAppB in plaintext.

Step-4. The clientAppB verifies that the RW sets received from E1b and E1c are same.

Step-5. The clientAppB submits the TX with a hash of the private data to the ODS.

Step-6. The ODS packs the TX in the latest block. The block with the hashed value is distributed to all the peers on Ch1.

Step-7. All the peers on the channel validate TX with the hash of the private data in a consistent way, without knowing the actual private data.

Step-8. Ledger update event is generated, and the clientAppB is notified.

\subsection{Reward Mechanism}

PrivySharing incentivizes the users to share their data with other users, stakeholders, or third parties by rewarding them with a local digital token named "PrivyCoin", as exhibited in Figure-6. PrivyCoin is just like an asset in the smart city network that is issued only by the network admin (Ministry of Development and Smart Services) against the payment in terms of fiat currency. The secure execution of such a TX is not covered in this paper. However, it is envisaged that the stakeholders can pay the ministry through any secure payment app and then receive the coins in their wallet, just like any other cryptocurrency/token. 
PrivyCoin is primarily used for trading or getting access to the data assets. After acquiring PrivyCoins, the stakeholder forwards the request for data access along with asset ID and the duration of access (in terms of days). Currently, in PrivySharing, the third parties/stakeholders pay one PrivyCoin to a user to get access to a data asset for one day (24 hours). Hence, if a stakeholder wants to get access to two data assets of a user for five days, he has to pay ten PrivyCoins to the user. Upon receiving the request to share data, it is only the prerogative of the data owner to initiate the data sharing TX. The data owner gets the incentive as soon as the data sharing TX is committed. In this context, if a stakeholder does not have requisite coins in his account, the TX will fail (shown in Figure-7). The pseudocode for the reward-based data sharing TX is illustrated in Algorithm-1. This algorithm can be summarized into four steps. Firstly, the data asset to be shared is obtained from the asset registry. Whereas, the input data structure of the data sharing $T X$ contains the asset type (e.g., Heart Rate, Blood Sugar, etc.), the asset reference (ID of the asset), the time duration of sharing (e.g., three days) and a list of stakeholders (e.g., P2, P4). Then, the algorithm checks whether the asset has already been shared with the stakeholders or not. After that, stakeholders pay PrivyCoins to the 420 data owner. Finally, the asset status is updated, and an event is emitted to notify the related parties, i.e., user and the stakeholders.

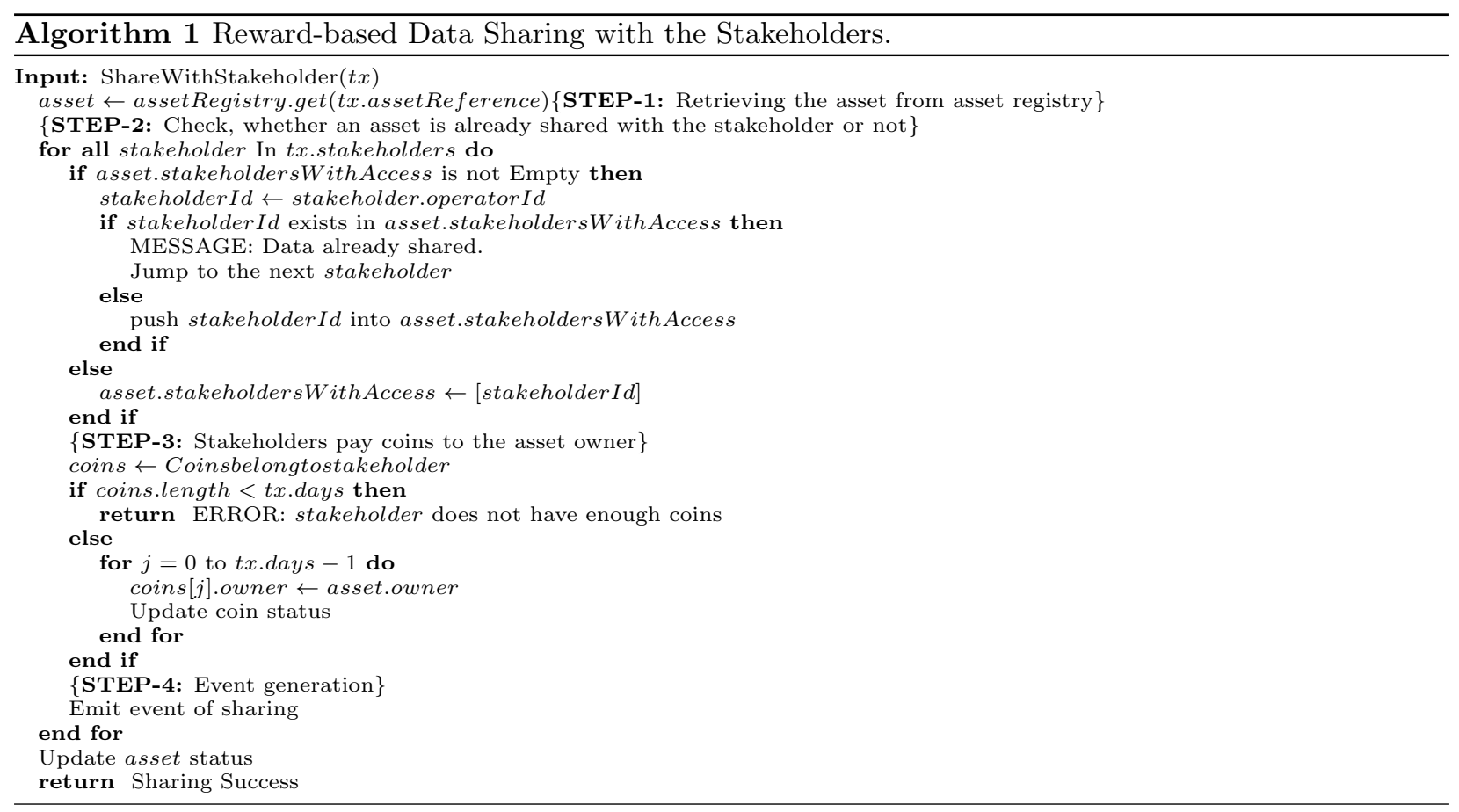

\section{Security Analysis}

The security, being the core objective of this work has been assessed at every level of the network operation. The key aspects shown in Figure-8 are illustrated as under.

425 When the blockchain network is first created, all the peers and orderer organizations are issued with certificates from respective RCA, or other trusted CAs. Then, a connection profile is created for all the network entities including Chs, ODS, organizations, peers, and CAs. The connection profile defines the complete blockchain network setup. E.g., for a $\mathrm{Ch}$, it defines the $\mathrm{Ch}$ name, its associated ODS and peers. It also defines which peers are the endorsing peers for that particular Ch. For an organization, it defines the namespace, ${ }_{430}$ MSP ID, member peers, and the respective CA. The peers' profile includes the namespace, URL including the port number, and the Transport Layer Security (TLS) certificate for its principal organization. The key point here is that no other peer (with the intention of endorsing the TXs on a $\mathrm{Ch}$ ) can join the network if it is not defined in the connection profile. It is clarified that by peers, we mean committing, endorsing or ODS 


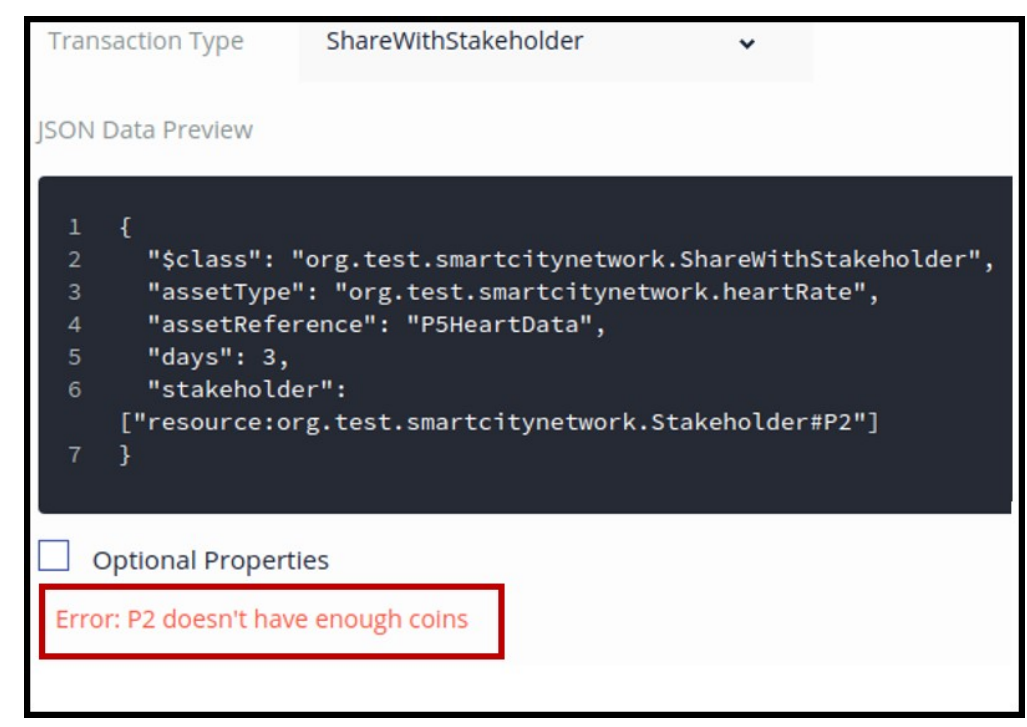

Figure 7: Error for not having enough coins

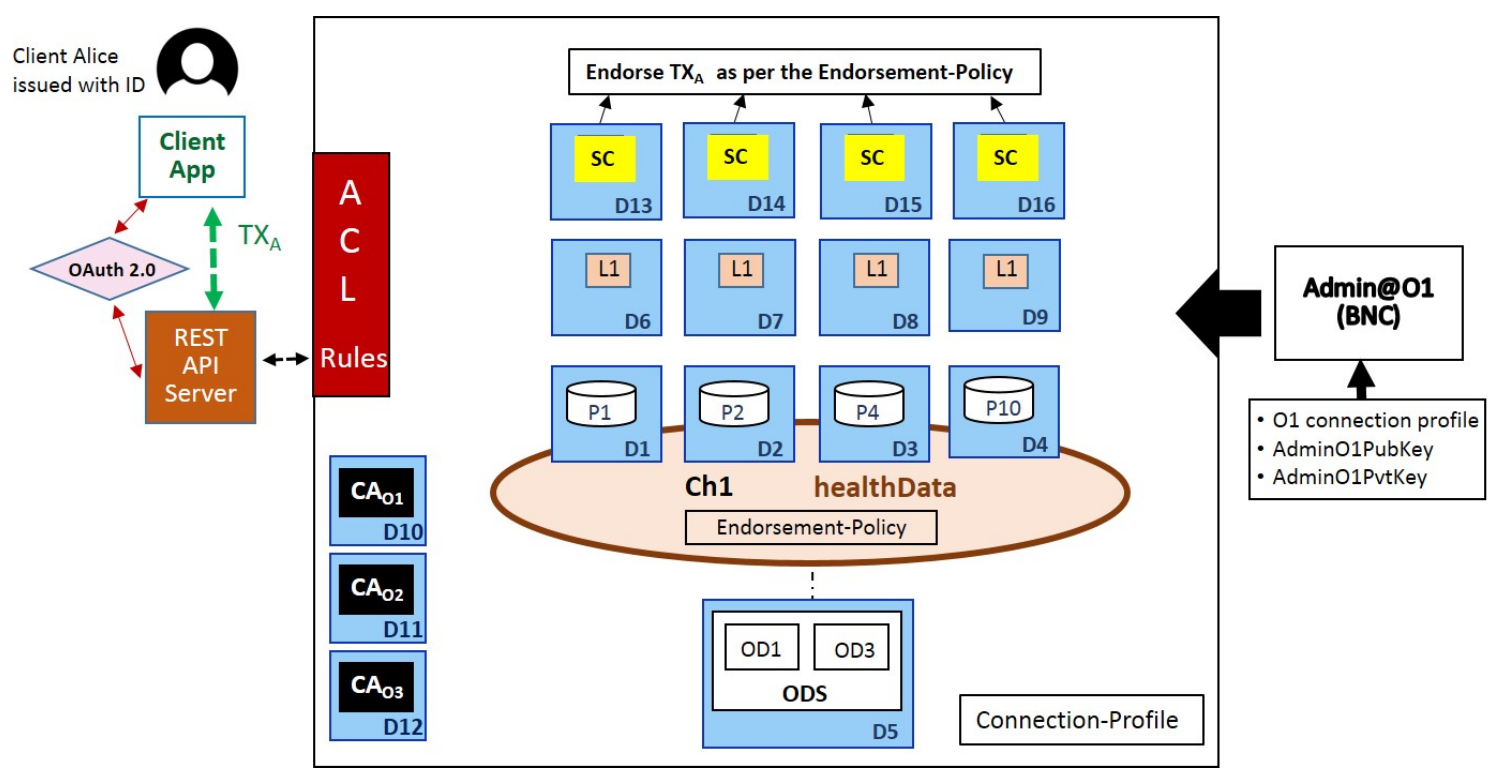

Figure 8: Elements of PrivySharing network security

peer nodes that maintain the blockchain network. Whereas, the users/clients access the blockchain network through REST API or clientApps. The smart city blockchain network entities including ODS, peers, CAs, ledgers, and SCs run in separate docker containers (symbolize by blue boxes numbered from D1 to D15 in Figure-8. This separation minimizes the effects of a container compromise, i.e., if one container's security is breached the other containers remain unaffected.

To deploy the business network model (PrivySharing in this case) that comprises asset definitions, TX 440 and event logic, and ACL rules on the blockchain, the admin of responsible organization (O1 in this scenario) requires a Business Network Card (BNC). The BNC is created using the connection profile of the organization and the valid public and private key for that admin issued by the authorized CA, as defined in the connection profile. The TXs initiated by the clientApps on a specific Ch are endorsed as per the endorsement 
policy defined for the respective Ch before the start of the business network. The endorsement policy may 445 include, e.g., what all peers (with endorsing ability) are required to endorse a TX on a Ch concerning health data. Similarly, a TX is considered valid, only if the response of all the required endorsing peers is the same. Hence, only a valid TX will update the world state. Another vital security feature of PrivySharing is that before the start of the business network on the blockchain, business network admins have to be defined and issued with the certificates (Public and Private key pairs) by the respective CAs. These certificates are later used to create the BNCs for the said admins to access the business network. Without a valid BNC, no one can add participants (clients/peers) for an organization. Moreover, every new client/peer added under an organization is also issued with an ID by the respective CA with the approval of the business network admin. These IDs are further used to control access to the users' profile and assets as per the ACL rules defined for the specific Ch.

455 As far as privacy of user data is concerned, the use of data specific Chs, private data collection, and data encryption does provide some degree of data privacy. However, even if a user's IoT device data is encrypted, still a passive network attacker can infer a pattern of user's activities. The same has been demonstrated by the researchers in 41. The authors exhibited that an adversary capable of monitoring the network traffic between a smart home gateway device and the internet can determine the type of IoT devices being used ${ }_{460}$ inside a smart home, based on DNS queries. Also, the attacker can analyze the metadata of the network traffic and observe variations in the IoT data send/receive rates. Hence, based on these abrupt changes in data rate/packet size, the adversary can deduce vital information about user's behavior and daily routine. Although, the conventional IoT classification methods do not apply to the blockchain, as the TXs in blockchain contain public keys instead of IP addresses, and are broadcast to the network. Nevertheless, to 465 avert the effects of malicious network traffic monitoring measures such as incorporation of VPN tunneling or obfuscating and shaping all smart home network traffic can be taken to mask variations that encode realworld behavior of the device owner.

Correspondingly, in blockchain-based IoT systems, the combination of device classification and user deanonymization can infer private information about a user to an adversary. Although, in PrivySharing, the IDs of all the members of the network are known and there is also a provision that each user can be issued with multiple cryptographic IDs (Public-Private key pairs) 42. Hence, users can use a different ID to communicate with every stakeholder. Such an arrangement seems robust against linking attacks [43]. However, blockchain researchers in [44 established the possibility of IoT devices classification by analyzing IoT device data stored on the blockchain by applying Machine Learning (ML) algorithms. Unlike in 41, an adversary is assumed to have access only to the data stored on the blockchain rather than the network traffic [44. The attack methodology identifies the IoT devices based on different patterns of timestamp differences in successive TXs of each type of device. However, researchers also proposed combinations of various methods of timestamp obfuscation to avoid device classification. These techniques include: introducing a random delay in the TXs of a device, combining multiple data packets of a specific device into a single TX, and lastly, 480 merging ledgers of numerous devices.

\subsection{ACL Rules}

PrivySharing has embedded user-defined ACL rules in the data sharing chaincodes to protect user data. The graphical illustration of the access control process based on some of the ACL rules is shown in Figure-9. These rules enforce that the data asset owners have access to their assets only, i.e., no user can see data 485 assets of any other user, and only the data owners can initiate a TX to share their data assets with other users/stakeholders. Similarly, a data owner has the right to revoke the sharing of his assets, and he can also delete his assets when no longer required without affecting the TX history stored on the blockchain. Moreover, as all the TXs are recorded on the blockchain, hence, to increase privacy, a data owner can see the TX history concerning his own assets only. Additionally, valid users can read and update their profiles only, and other users/stakeholders cannot see each other's profile. Users can also delegate the stakeholders to create assets on their behalf. E.g., Alice (P5) delegates her primary medical center (P2) to create a health data asset for her. Accordingly, the stakeholders can only see the data assets that are shared with them or created by them. Lastly, all the users/stakeholders can view their coins only. The pseudocode of the data asset unsharing and asset deletion is accordingly shown as Algorithm-2, and Algorithm-3, respectively. 


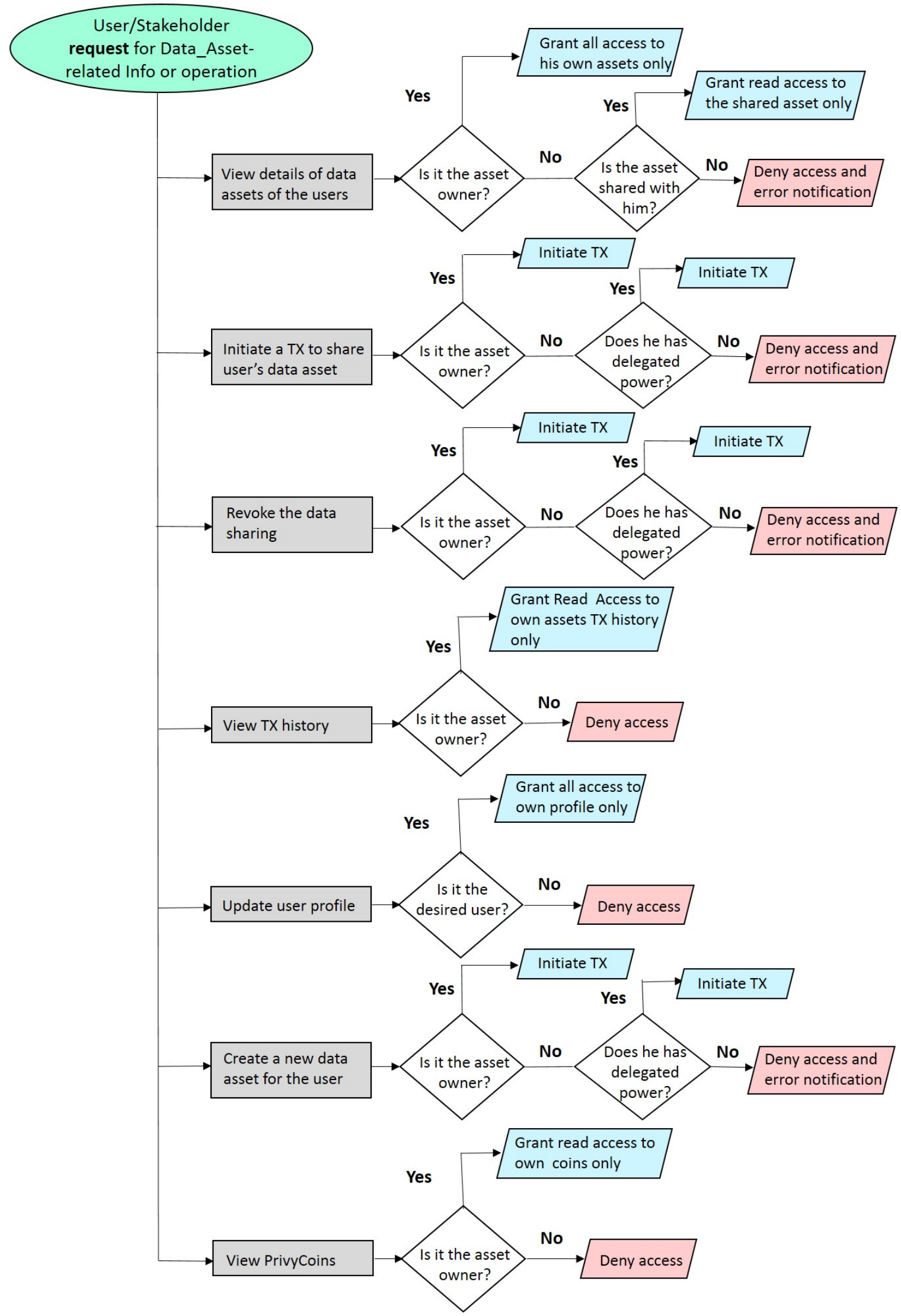

Figure 9: ACL rules 

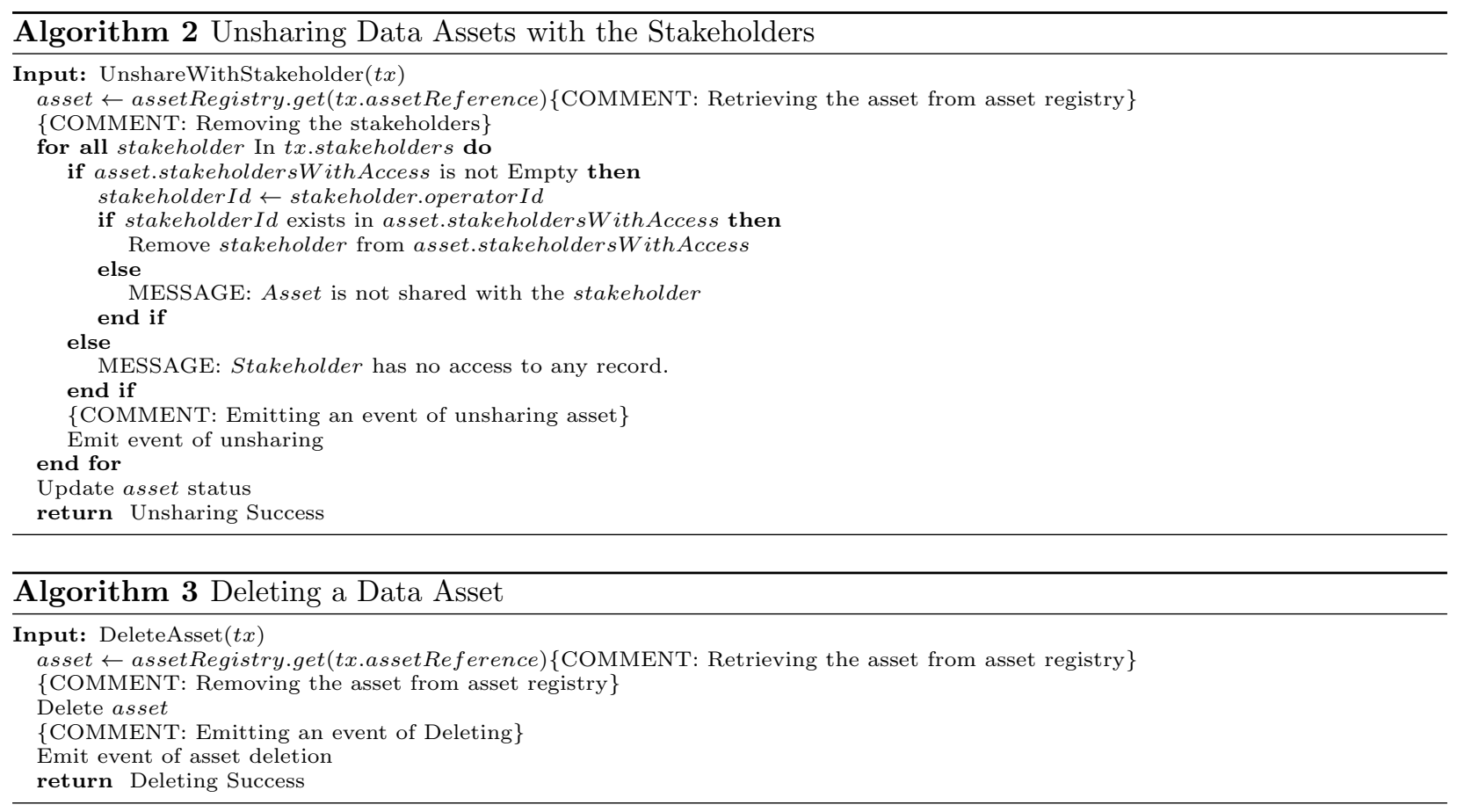

\subsection{Security of REST API and Dapp}

Access to the REST API is secured using the API key which is required to launch the REST API. In addition to the API Key, OAuth-2.0 authorization protocol 45] is also employed to authorize access to PrivySharing REST server instance, and allow the end-users/clients to interact with the PrivySharing business network deployed on the blockchain. The mechanism of OAuth-based REST API security protocol 500 is shown in Figure-10. In step-1, the client/user/third-party App sends an authorization request to the PrivySharing business network admin from O1 that also acts as the resource owner. The resource owner then replies with the authorization grant. In step-3, the client sends an authorization token request containing the authorization grant received from the resource owner in step- 2 to the authorization server. After validating the authorization grant, the authorization server issues an access token to the client. The client then requests the PrivySharing REST Server to grant access by presenting the access token. Finally, in step-6, if the token is valid, the client is granted access to call the PrivySharing REST API operations. Currently, there are more than three hundred options for the client REST Server authentication strategies including SAML, LDAP, GitHub and a blend of OSN such as Facebook and Google. For this PoC, we have used Passport-GitHub strategy to authenticate the users. The detailed procedure of enabling OAuth for PrivySharing REST Server is depicted in Figure-11.

Furthermore, due to the distributed nature of the SCs, the integrity of any business network deployed on the blockchain is guaranteed. Similarly, it also protects against hacking of servers, where, the attackers can change the policy rules, escalate access rights, etc. Correspondingly, protection against application and web vulnerabilities can also be guaranteed with high probability, as any change in the smart contract requires installing and instantiating a new version of the contract on all the endorsing peers. However, it cannot be done discretely. Additionally, due to a distinction between blockchain and the world state, an auditable log of TXs and events is maintained without compromising the privacy of the users' data.

\subsection{Restricted Access to User Data Assets via Multiple Chs}

In addition to restricting access to users' data assets through ACL rules within a Ch, the use of data specific Chs is also helpful in preserving users' data privacy. Through our PoC, we have validated that every $\mathrm{Ch}$ in PrivySharing smart city network is independent of other Chs with associated Ch members. As shown in Figure-12, when P13 from O7 (not a member of Ch1), tries to query a user's heartRate data, he gets an access 


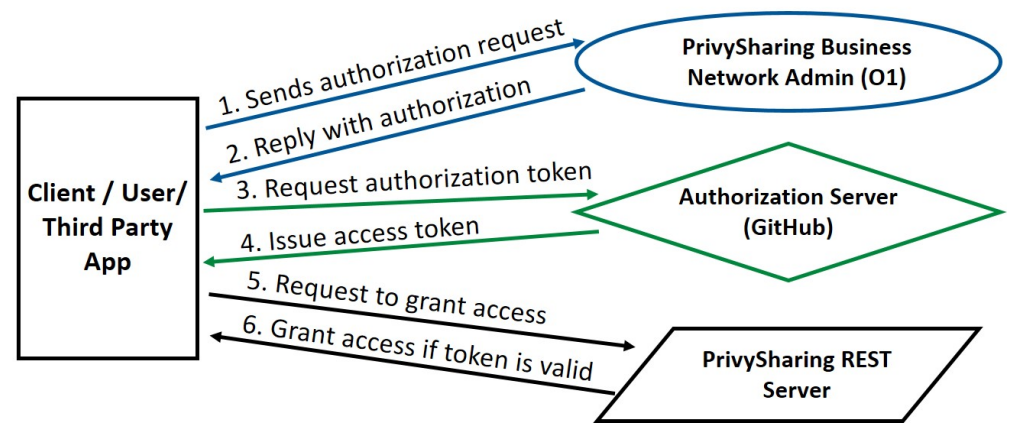

Figure 10: PrivySharing REST server OAuth protocol

denied error because he is not authorized to access any data asset propagated on Ch1. As PrivySharing is a permissioned consortium blockchain, all the network members are duly registered and authenticated before joining the network. However, even if an unauthorized node gets added to the system through a corrupt network admin, the ACL rules prohibit the intruder from unauthorized access to users' data assets.

Moreover, Table-3 shows the methodology we adopted to achieve the security objectives derived from smart city threat environment and EU GDPR requirements. However, one of these objectives, i.e., IoT device integrity check has not been addressed in this paper.

\subsection{Validation of ACL Rules}

The validity of the ACL rules was checked on both, the Hyperledger Composer-Playground and the REST API. E.g., As shown in Figure-14a and b, to compare the access rights we have created a user with admin ${ }_{50}$ rights that can view assets (blood alcohol level) of all the users, i.e., P5 and P6 in this case. Whereas, the user P5 with ID Pid5 can only see his assets. Moreover, Figure-14k and d show that initially, a user P4 with id Pid4 cannot see any asset, as no asset is currently shared with him. However, once user P5 shares his blood alcohol level with P4, he can then see P5's blood-alcohol level. Similarly, only P5 can initiate a TX to share its assets. Whereas, if $\mathrm{P} 4$ tries to share the asset of P5 with any other entity then he will get an error (as shown in Figure-15) as he currently does not have the right to initiate a data sharing TX. As far as the purging of a data asset is concerned, as shown in Figure-16a, a data asset say P5's blood sugar can be deleted. However, Figure-16b manifests that the historical record (TX history) of a deleted asset remains 


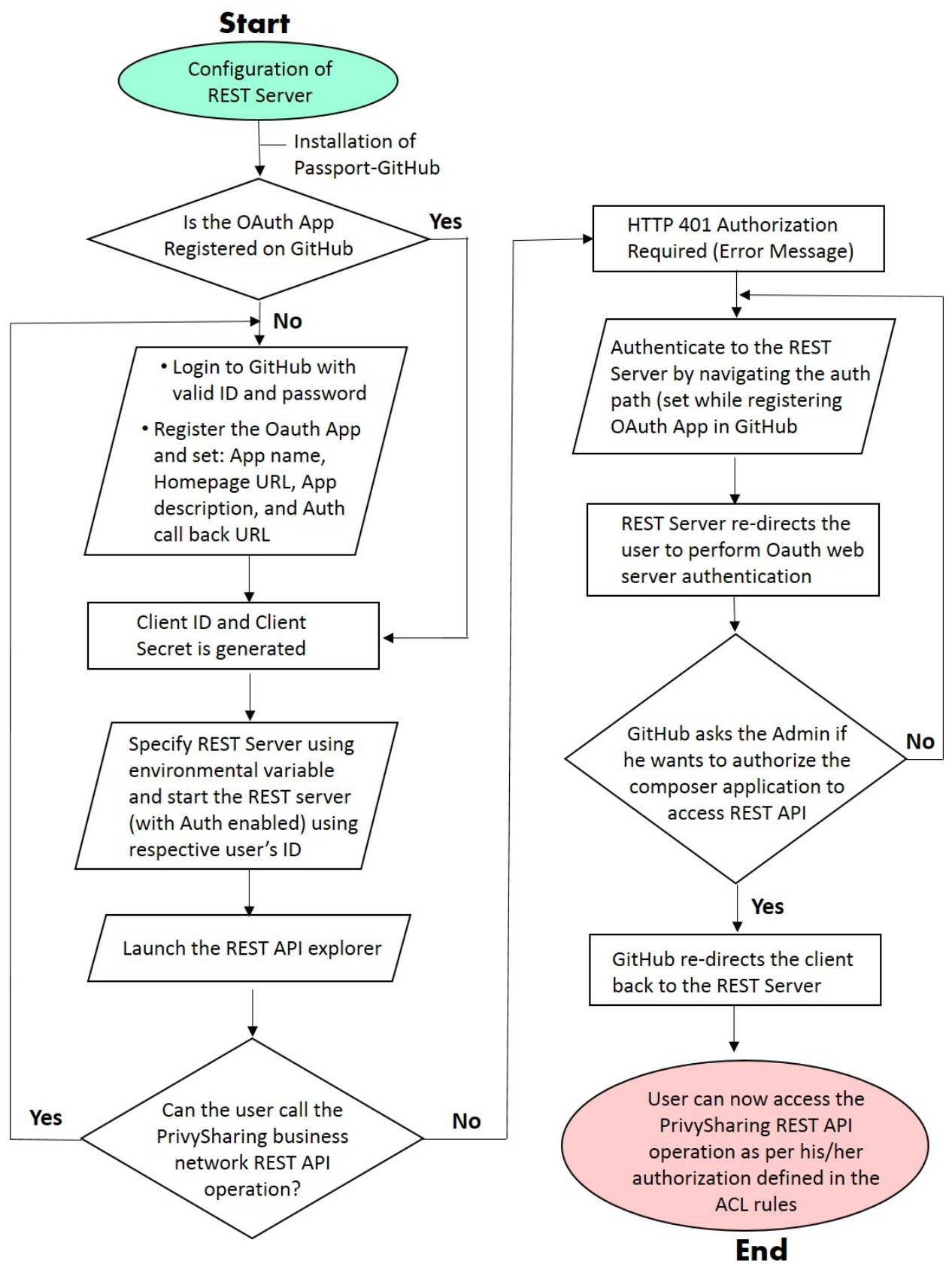

Figure 11: PrivySharing REST server OAuth flowchart 
Table 3: Methodology to Achieve PrivySharing Objectives

\begin{tabular}{|c|c|c|c|}
\hline Ser & $\begin{array}{l}\text { Factors Deriving the Ob- } \\
\text { jectives }\end{array}$ & Objectives & Methodology \\
\hline \multicolumn{4}{|c|}{ Threats to User/Data Security in a Smart City Environment } \\
\hline 1. & User privacy (ID disclosure) & $\begin{array}{l}\text { Reduce the possibility of users' real world ID disclo- } \\
\text { sure }\end{array}$ & $\begin{array}{l}\text { PKI (X.509 Certificates) based mul- } \\
\text { tiple IDs for users }\end{array}$ \\
\hline 2. & User data privacy & $\begin{array}{l}\text { Data confidentiality at rest and in transit, prevent over } \\
\text { data collection, controlled access to data as defined by } \\
\text { the data owner }\end{array}$ & $\begin{array}{l}\text { Data encryption, use of SSL/TLS for } \\
\text { data security in transit, user-defined } \\
\text { ACL rules, use of multiple Chs and } \\
\text { private data collection within a Ch }\end{array}$ \\
\hline 3. & $\begin{array}{l}\text { Single point of failure (from } \\
\text { physical as well as trust } \\
\text { point of view) }\end{array}$ & Distributed data storage and decentralized control & Hyperledger-Fabric Blockchain \\
\hline 4. & False injection of data & Prevent data injection by unauthorized users & $\begin{array}{l}\text { ID management, authentication and } \\
\text { participation of only authorized } \\
\text { nodes in the network. Moreover, TX } \\
\text { initiation rights given to data owners } \\
\text { or the parties given delegated powers } \\
\text { by the data owners }\end{array}$ \\
\hline 5. & Vulnerability to Sybil Attack & Prevent Sybil Attack & $\begin{array}{l}\text { User ID management and TX initi- } \\
\text { ation by authorized entities only as } \\
\text { per ACL rules }\end{array}$ \\
\hline 6. & $\begin{array}{l}\text { Lack of common security } \\
\text { framework for heterogeneous } \\
\text { IoT devices with different } \\
\text { communication protocols } \\
\text { and diverse hardware pa- } \\
\text { rameters }\end{array}$ & $\begin{array}{l}\text { Provide a common platform to store data transmit- } \\
\text { ted/received from the heterogeneous sensors, irrespec- } \\
\text { tive of their diverse hardware and communication tech- } \\
\text { nologies }\end{array}$ & Hyperledger-Fabric Blockchain \\
\hline 7 . & $\begin{array}{l}\text { Threats to data integrity } \\
\text { (data forgery and manipula- } \\
\text { tion) }\end{array}$ & Preserve user data integrity & $\begin{array}{l}\text { User authentication and restricted } \\
\text { privileges to update user data, and } \\
\text { blockchain's inherent data integrity } \\
\text { protection }\end{array}$ \\
\hline 8. & $\begin{array}{l}\text { Threats to smart city appli- } \\
\text { cations }\end{array}$ & $\begin{array}{l}\text { Protect applications against the escalation of privi- } \\
\text { leges and alteration attacks }\end{array}$ & Use of smart contracts based DApps \\
\hline 10. & Scalability & Contain the size of the blockchain & $\begin{array}{l}\text { Use of blockchain to store TX logs } \\
\text { only, whereas a world state is used } \\
\text { to store user data updated states }\end{array}$ \\
\hline 11. & $\begin{array}{l}\text { TX Latency and Through- } \\
\text { put }\end{array}$ & More TX throughput with less latency & $\begin{array}{l}\text { Use of multi-Ch blockchain as com- } \\
\text { pared to a single-Ch blockchain }\end{array}$ \\
\hline \multicolumn{4}{|c|}{ Essential GDPR Requirements for User Data Security } \\
\hline 1. & $\begin{array}{l}\text { Personal data to be pro- } \\
\text { cessed only with data } \\
\text { owner's consent }\end{array}$ & $\begin{array}{l}\text { The data owner is in complete control of his data, } \\
\text { transparency of the complete process, visibility of all } \\
\text { security and data access control changes }\end{array}$ & $\begin{array}{l}\text { Chaincode-based user data access } \\
\text { control rules, maintaining, and dis- } \\
\text { seminating TX log on the need to } \\
\text { know basis (Only a data owner or an } \\
\text { authorized entity can see the TX log } \\
\text { of a specific asset) and data sharing } \\
\text { TX can only be initiated by the data } \\
\text { owner }\end{array}$ \\
\hline 2. & Privacy by design & $\begin{array}{l}\text { By default user data should be inaccessible to all, ex- } \\
\text { cept those who are specifically allowed by the data } \\
\text { owner }\end{array}$ & $\begin{array}{l}\text { Access control rules deny everyone to } \\
\text { see other's profile and assets unless } \\
\text { explicitly shared by the data owner }\end{array}$ \\
\hline 3. & $\begin{array}{l}\text { Commissioned data process- } \\
\text { ing (i.e., data collection and } \\
\text { processing as per the con- } \\
\text { tract between the data owner } \\
\text { and other parties) }\end{array}$ & $\begin{array}{l}\text { A contract-based user data sharing that should con- } \\
\text { form to the contractual obligations }\end{array}$ & $\begin{array}{l}\text { Business logic is transformed into } \\
\text { Smart Contracts for secure and effi- } \\
\text { cient data sharing as per contractual } \\
\text { obligations }\end{array}$ \\
\hline 4. & $\begin{array}{l}\text { Data owner should have ac- } \\
\text { cess to all the information } \\
\text { concerning his data (i.e., } \\
\text { where is it stored, who has } \\
\text { access to it, and for how } \\
\text { long) }\end{array}$ & $\begin{array}{l}\text { A transparent system, where data owner has complete } \\
\text { visibility of the process and should be able to see and } \\
\text { control the access to his data }\end{array}$ & $\begin{array}{l}\text { User-defined data access control, and } \\
\text { TX log management }\end{array}$ \\
\hline 5. & $\begin{array}{l}\text { Right to forget, i.e., user } \\
\text { data to be erased when no } \\
\text { longer required }\end{array}$ & $\begin{array}{l}\text { The system should allow user data deletion after a spe- } \\
\text { cific time, when the contract between the user and a } \\
\text { third party expires, or when data is outdated or no } \\
\text { longer required. Hence, there should be some distinc- } \\
\text { tion between TX log maintenance and user data stor- } \\
\text { age. Such that even if user data is deleted, we are still } \\
\text { able to verify the integrity of the past data }\end{array}$ & $\begin{array}{l}\text { The world state is distinct from the } \\
\text { blockchain. Hence, data/asset owner } \\
\text { can delete user data from the world } \\
\text { state without affecting TX log his- } \\
\text { tory }\end{array}$ \\
\hline 6. & Transparency & $\begin{array}{l}\text { The system should be transparent, i.e., log all the ac- } \\
\text { tivities concerning users' data (when and who modi- } \\
\text { fied the access control policies for data and updated } \\
\text { the data itself) }\end{array}$ & $\begin{array}{l}\text { TX log management and event noti- } \\
\text { fication }\end{array}$ \\
\hline
\end{tabular}


Figure 12: Access denied for out of Ch data query

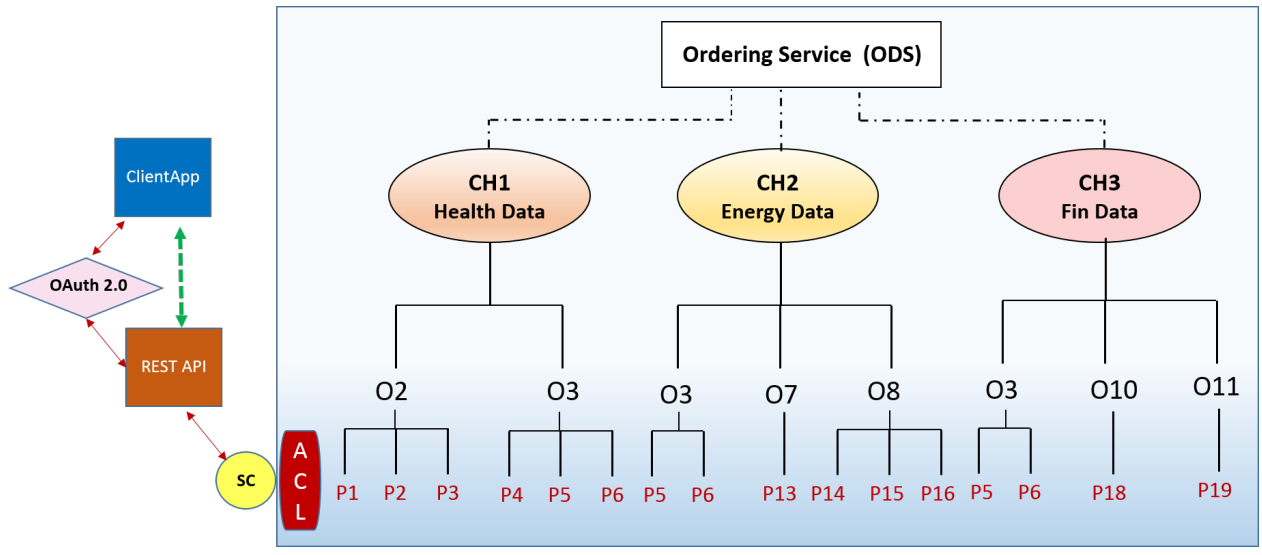

Figure 13: Experimental Settings Phase-1

immutable in the blockchain. Sequel to this, the TX history concerning the data assets can only be viewed by respective users only. As shown in Figure-16c and d, only P5 (Alice) can view the record of her data sharing TXs. Whereas, any other user, say P6 (Bob) cannot see Alice's TX history. However, even if a blockchain admin is allowed to view the transaction history of all the nodes for accountability, the admin still cannot see the value of the data asset being shared.

\subsection{Performance Efficiency}

Though, a detailed comparison of performance efficiency of Hyperledger Fabric with some of its counterparts is already presented in 21 and [46. However, as per the experimental settings for phase-1 (as shown in Figure-13), we measured the time taken to commit various types of TXs in the preview of PrivySharing. The average commit time has been measured for three different TXs based on ten iterations. The TXs include; plain text (PlainText) TX, private data (PvtData) TX, and encrypted private data (EncPvtData) TX. These TXs are analyzed in two different consensus environments, i.e., SOLO and Kafka.

It is evident from Figure-17 that all types of TXs irrespective of the employment methodology take less than 490 (milliseconds)ms to commit in a new block. However, there is a clear pattern that the EncPvtData TXs for both asset generation and sharing take more time to commit than the PvtData and PlainText TXs. Moreover, the time taken by an asset sharing TX is lower than the asset generation/creation TX in almost all three cases. Similarly, Figure-18 highlights the average (avg) time taken for state validation, block commit, and state commit for asset generation and asset sharing TXs with SOLO and Kafka consensus both. It can be ascertained that the time taken for block commit (represented by rust strip) in all three cases, i.e., EncPvtData, PvtData, and PlainText TXs, does not show many variations. However, the state commit time (expressed in the grey strip) significantly reduces for the PlainText TXs with SOLO and Kafka consensus in both cases, i.e., asset generation and asset sharing TXs. Similarly, the overall TX commit time for a plain text TX is lower than the EncPvtData and PvtData TXs.

In the second phase of the experiment, we measured various performance indicators of PrivySharing using Hyperledger Caliper as per the settings shown in Table-4. For the initial test, we ran thirty rounds of the experiment for both one-Ch and three-Ch scenarios with Kafka ordering service (consensus). There were six peers and six clients operating in the one-Ch and two peers and two clients per Ch in the three-Ch scenario. Total three hundred TXs were input to the system at the rate of fifty tps (Transactions per second), in both scenarios. The highlight of this experiment as shown in Figure-19 is that the three Ch scenario 


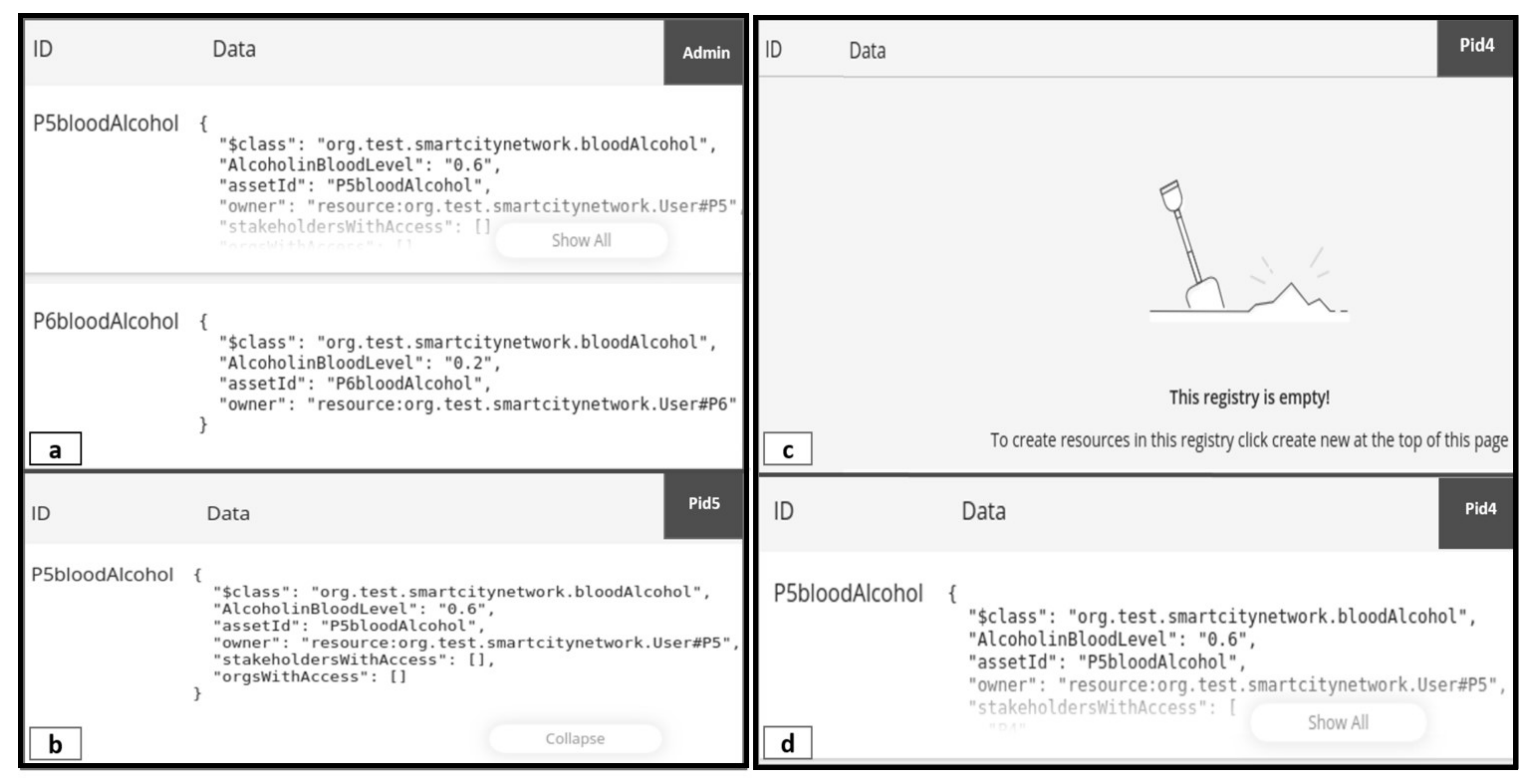

Figure 14: Validation of assets access control

Table 4: Experimental Settings Phase-2

\begin{tabular}{|l|l|l|}
\hline \multicolumn{1}{|c|}{ Parameters } & $\begin{array}{l}\text { Settings for One CH } \\
\text { Scenario }\end{array}$ & $\begin{array}{l}\text { Settings for Three } \\
\text { CHs Scenario }\end{array}$ \\
\hline Number of Chs & 01 & 03 \\
\hline Number of Input TXs & 300 & 300 \\
\hline TX Send Rate & 50 tps & 50 tps \\
\hline Number of Member Organizations & 6 & 6 \\
\hline Peers Per Ch & 6 & 2 \\
\hline Total Peers & 6 & 6 \\
\hline Number of Orderer Nodes & 4 Kafka Nodes & 4 Kafka Nodes \\
& 3 Zookeeper Nodes & 3 Zookeeper Nodes \\
\hline Number of Clients & 6 & 6 \\
\hline Number of Experiment Rounds & 30 & 30 \\
\hline
\end{tabular}

has demonstrated efficient performance contrary to the single Ch scenario, with an avg throughput of 42.4 tps and avg latency of $1.54 \mathrm{sec}$ at the TX Send Rate of 50 tps. After this primitive comparison, we also determined the p-values [47, 48, for both the scenarios to substantiate our findings. In that, we first ap590 plied independent two-sample T-test on latency measurements to determine the p-value to accept or reject the null hypothesis, i.e., "The average latency of the one-Ch network is equal to the average latency of the three-Ch network." Whereas, the alternative hypothesis is; "The average latency of the one-Ch network is greater than the average latency of the three-Ch network." The p-value resulted from the first test on system latency was $8.62 \times 10^{-31}$, which is less than 0.05 . The result suggests the rejection of the null hypothesis in favor of the alternative hypothesis. Therefore, it is more probable that the average latency of the one-Ch network is higher than the average latency of the three-Ch system. Later, The second two-sample T-test was performed over throughput values. The null hypothesis in this case was; "The average throughput of the one-Ch network is equal to the average throughput of the three-Ch network." Whereas, the alternative hypothesis states that "The average throughput of the one-Ch network is less than the average throughput of the three-Ch network." The p-value emanated from this proceeding was $1.23 \times 10^{-28}$, which is smaller than 0.05. Hence, the result asserts the rejection of the null hypothesis in favor of the alternative hypothesis. Therefore, it is much likely that the average throughput of the one-Ch network is smaller than the average throughput of the three-Ch system. Hence, based upon the p-values, it can be concluded that the one-Ch network has inferior performance in terms of high latency and low throughput as compared to the three-Ch 


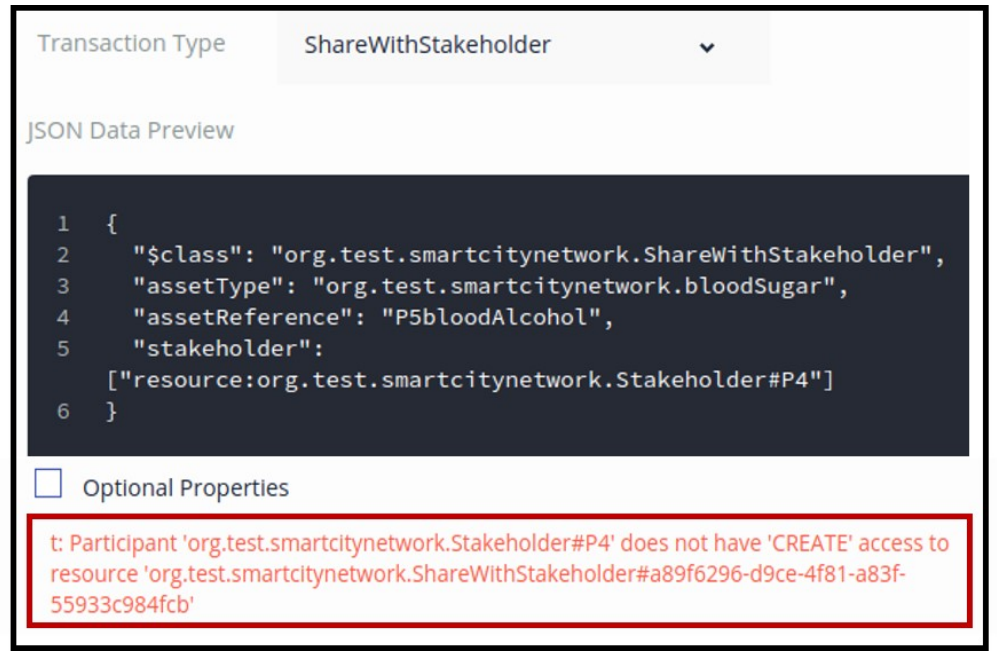

Figure 15: Validation of TX initiation rights

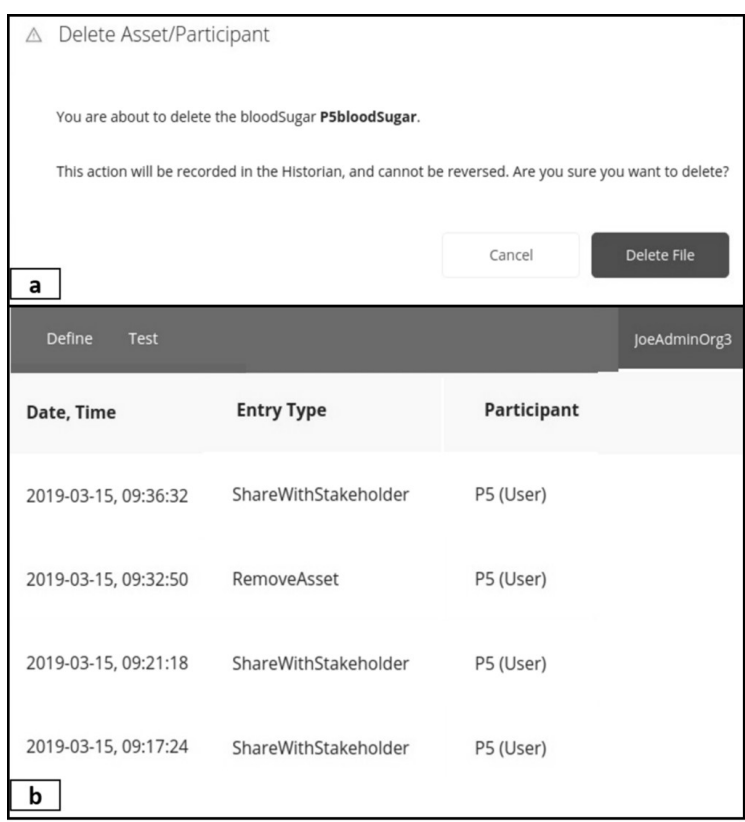

\begin{tabular}{|c|c|c|c|}
\hline Define Test & & & Pid5 \\
\hline Date, Time & Entry Type & Participant & \\
\hline 2019-03-15, 09:21:18 & ShareWithStakeholder & P5 (User) & view record \\
\hline 2019-03-15, 09:17:24 & ShareWithStakeholder & P5 (User) & view record \\
\hline 2019-03-15, 05:45:20 & ActivateCurrentldentity & none & view record \\
\hline $\begin{array}{l}2019-03-15,05: 43: 45 \\
\text { C }\end{array}$ & ActivateCurrentldentity & none & view record \\
\hline Define & & & Pid6 \\
\hline Date, Time & Entry Type & Participant & \\
\hline $2019-03-15,05: 45: 20$ & ActivateCurrentIdentity & none & view record \\
\hline $2019-03-15,05: 43: 45$ & ActivateCurrentIdentity & none & view record \\
\hline 2019-03-15, 05:35:09 & ActivateCurrentldentity & none & view record \\
\hline $\begin{array}{l}2019-03-15,05: 29: 18 \\
\text { d }\end{array}$ & ActivateCurrentldentity & none & view record \\
\hline
\end{tabular}

Figure 16: Historical record of purged data asset and visibility of TX history

605

network.

In the third phase of the performance testing, we mapped the correlation between different performance indicators for the three-Ch network. TX Send Rate was pitched against network latency and throughput, as per the test settings shown in Table-5. The experiment was run for ten rounds with varying TX Send Rate in each round. Although we had set specific TX Send Rate for the test case, however, the actual Send Rate to process and submit the TXs, respectively. Figure-20 a interprets the relationship between TX Send Rate and network latency. The avg latency increases uniformly until the TX Send Rate reaches around 106 tps. After that, the latency starts fluctuating between 3 and 4 secs. Correspondingly, Figure-20p also highlights a similar trend, in which the network throughput rises with the increase in the TX Send Rate. However, once 

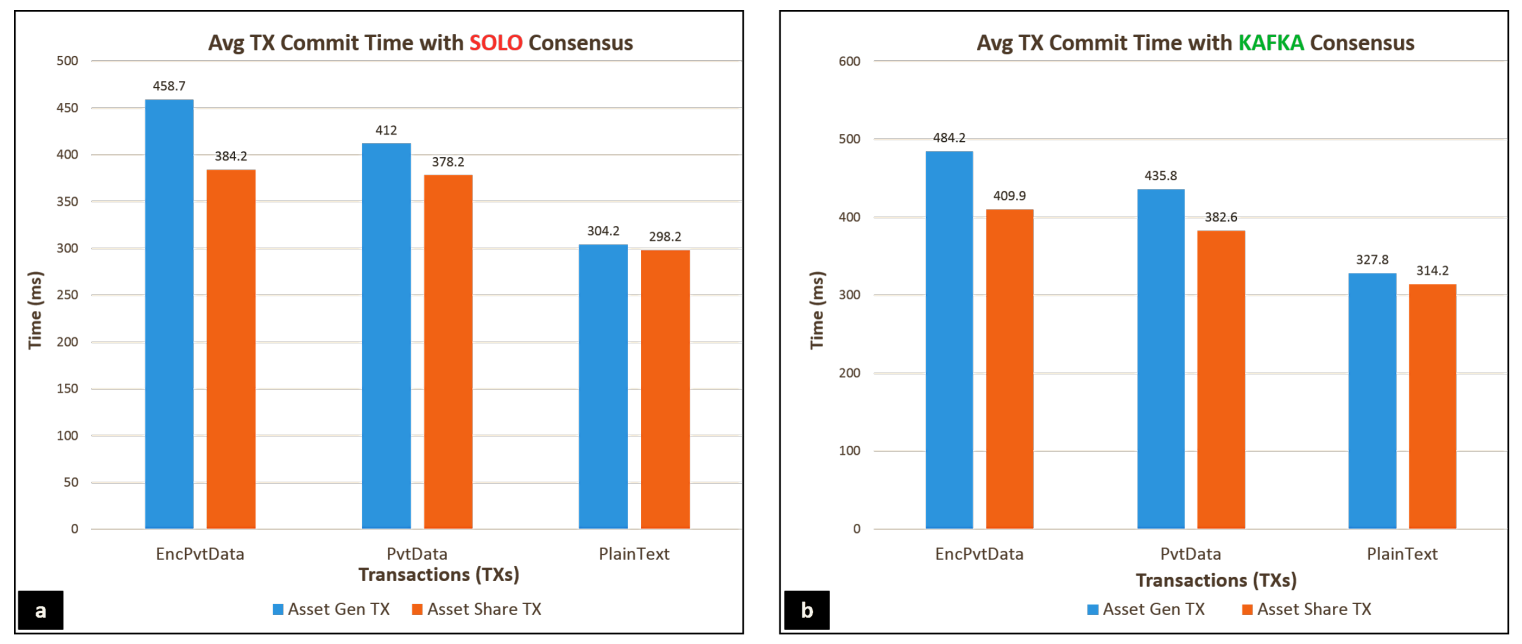

Figure 17: Avg TX Commit Time

Table 5: Experimental Settings Phase-3

\begin{tabular}{|l|l|}
\hline \multicolumn{1}{|c|}{ Parameter } & Settings \\
\hline Number of Chs & 03 \\
\hline Number of Input TXs & 300 \\
\hline $\begin{array}{l}\text { TX Send Rate (configured) for Ten } \\
\text { Rounds (tps) }\end{array}$ & $25,50,75,100,125,150,175,200,225,250$ \\
\hline $\begin{array}{l}\text { TX Send Rate (actual) for Ten Rounds } \\
\text { (tps) }\end{array}$ & $\begin{array}{l}24.4,47.4,70.9,89.6,106.4,116.3,145.8,154.3, \\
198.2,199.1\end{array}$ \\
\hline Number of Member Organizations & 6 \\
\hline Peers Per Ch & 2 \\
\hline Total Peers & 6 \\
\hline Number of Orderer Nodes & 4 Kafka Nodes \\
\hline Number of Clients Per Ch & 3 Zookeeper Nodes \\
\hline Total Clients & 2 \\
\hline Number of Experiment Rounds & 6 \\
\hline
\end{tabular}

TX Send Rate reaches 106, the throughput waffles between 50 and 56 tps. We believe that such a result is induced by the small number of orderer nodes, which could not handle more than 200 tps. Likewise, the latency in TX confirmation increases with the rise in TX Send Rate. Later, we also studied the correlation between an increase in the number of peers and avg latency, and throughput respectively at varying TX send rates (as shown in Figure-21a, b, and c). For this test, there were six clients, and the number of peers varied from 6 to 24 in an increment of 6 . It is observed that the throughput is mostly consistent with the send rate until the number of peers goes beyond 18. It can also be seen in Figure-21k that the throughput decreases notably as the number of peers reaches 24 . Similarly, the latency also increases with the increase in the number of peers. Such a behavior can be attributed to the number of endorsing and orderer nodes in the network that have to endorse and pack the TXs in the blocks and broadcast new blocks, respectively. Moreover, it can also be accredited to the fact that for this experiment all the peers were run on a single machine in a constrained environment. Hence, once in distributed setting, each peer is expected to perform much better. It is also believed that the TX throughput can be scaled by load balancing TX endorsement across a pool of endorsers [49].

The experimental results uphold the idea of a multi-Ch blockchain network, as the same has demon${ }_{630}$ strated more throughput and less latency than the one-Ch system. The network latency and throughput in Hyperledger-Fabric depend upon numerous factors, such as, application design, fabric network architecture, specifications of endorsement policies, complexities of ACL rules, application/chaincode language, number of endorsers and ordering nodes, the batch timeout, and the physical or the virtual network infrastructure 


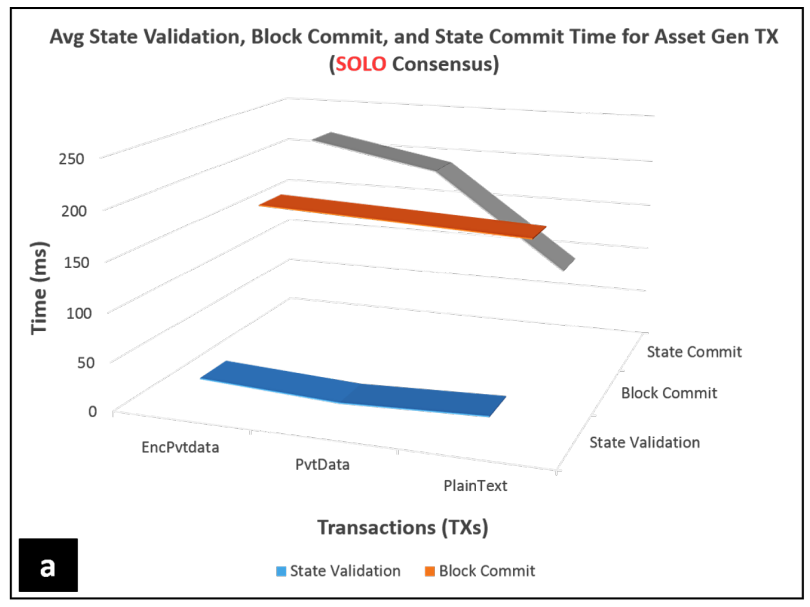

Avg State Validation, Block Commit, and State Commit Time for Asset Sharing TX (SOLO Consensus)

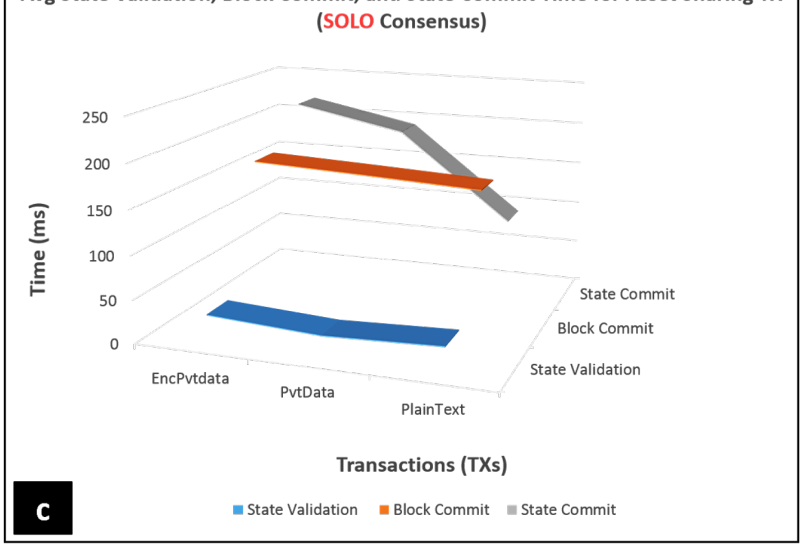

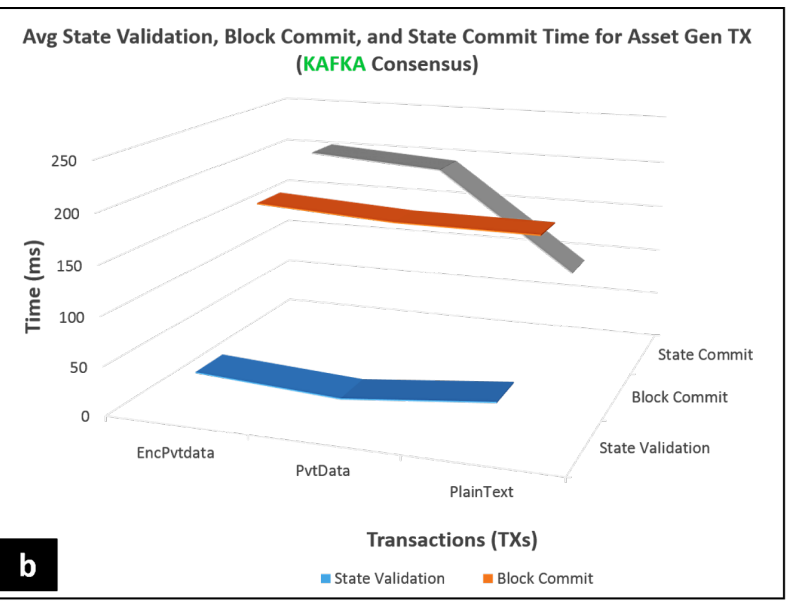

Avg State Validation, Block Commit, and State Commit Time for Asset Sharing TX

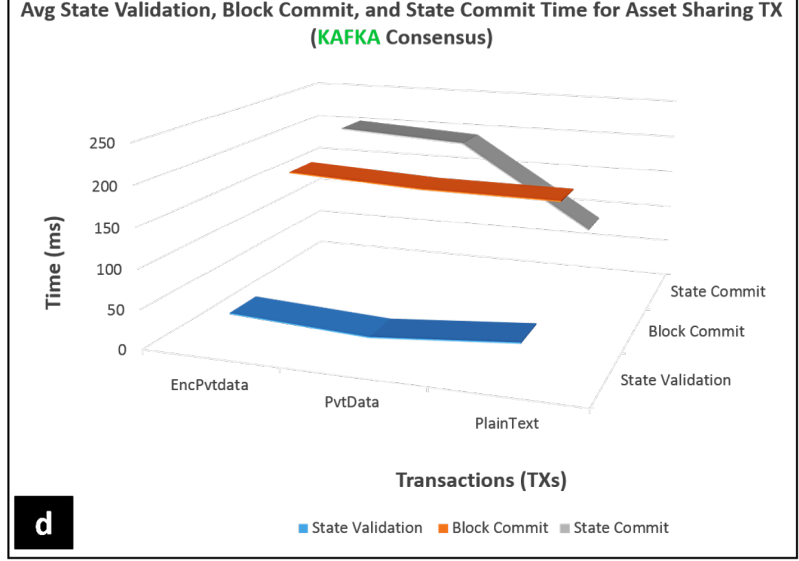

Figure 18: Comparison of State validation, Block commit, and State commit avg time

[49. Hence, a meticulously designed and laid out blockchain network and application can yield higher TX ${ }_{635}$ throughput with less latency. E.g., FabCoin built on top of Hyperledger-Fabric can achieve a throughput of over 3560 tps with Kafka ordering service [50].

\subsection{Limitations and Open Challenges}

\subsubsection{Multiple Ledger Storage by The Peers}

The use of multiple data specific Chs is presumed to be scalable than a single Ch. However, since committing peers have to maintain numerous ledgers, there may be a massive resource requirement for such nodes in a vast smart city network.

\subsubsection{IoT Device Integrity}

Electronic equipment, once connected to the internet is vulnerable to cyber-attacks. Resultantly, a hacker can hack into the electronic device and install malware, or modify the software or hardware components to alter the legitimate operation of the device [51, 52, 53. Moreover, if an electronic device is physically compromised, the attacker can also change the hardware components, i.e., extend device memory, increase RAM, increase or decrease processor speed, change network configuration, activate or deactivate unauthorized ports or interfaces (JTAG, UART etc), and change I/O (input/output) pins configuration [5, 54, 55]. Moreover, an electronic device malfunction can be caused by an unintentional or unprovoked technical fault, hardware or

software failure, and a human error. Existing methods of device integrity check are based on code or memory attestation. These methods do not protect against physical compromise of the device, and modification of 


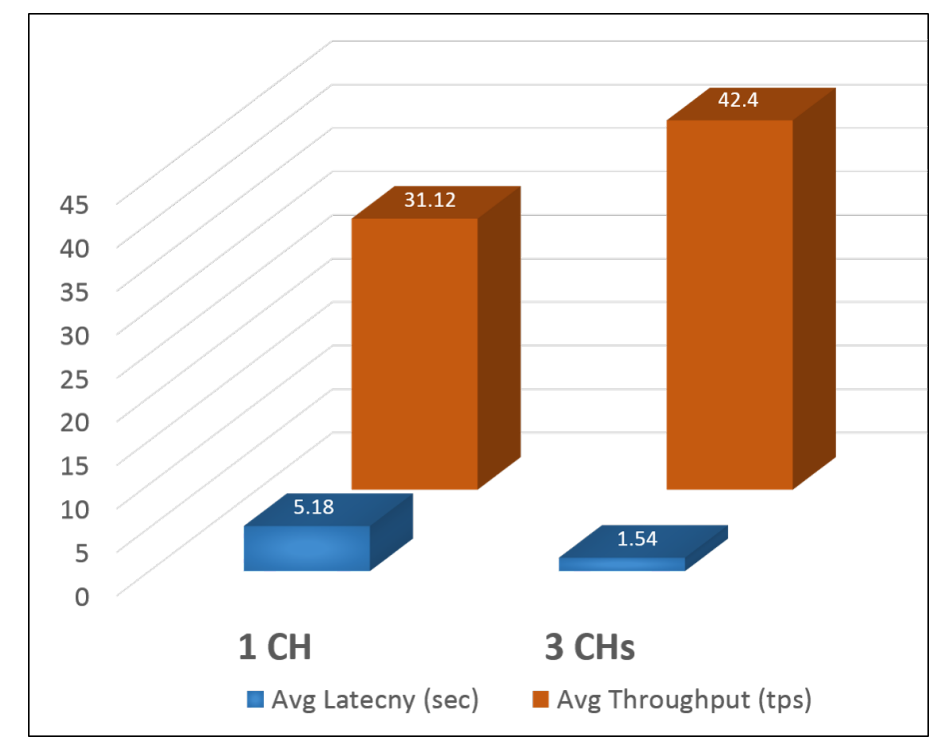

Figure 19: Comparison of Avg Latency and Avg Throughput in One-Ch and Three-Ch Scenario
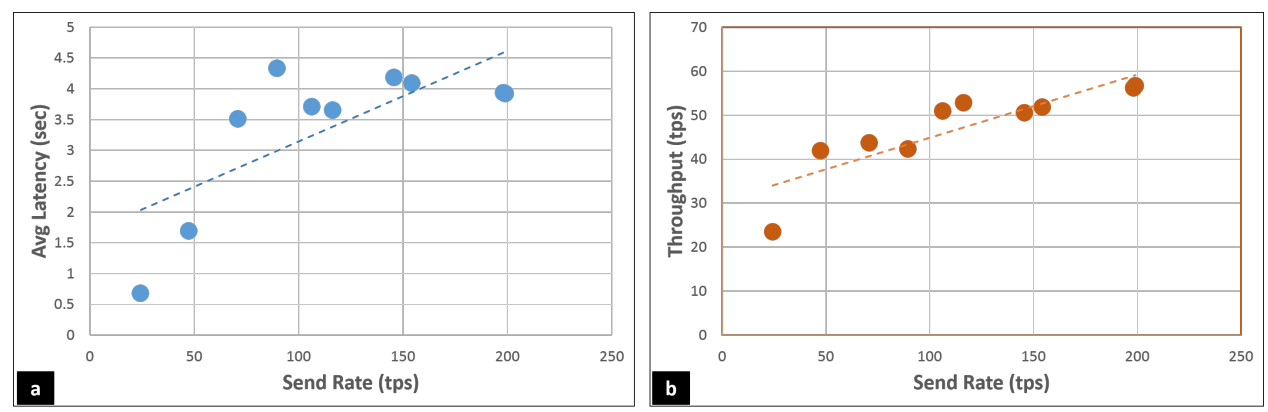

Figure 20: a) Correlation between TX send rate and latency. b) Relation between TX send rate and network throughput

hardware or software components 4. Contrarily, IoT data being the essential element to provide various seamless services in a smart city environment necessitate that the device initially generating and processing that data should be credible, i.e., only a legitimate and clean device should be able to input data to the blockchain. Whereas, currently, there is no plausible mechanism to test the integrity of the IoT devices at run time.

\subsection{A Way Forward to Address the Limitations}

\subsubsection{Alternative to Ledger Storage by the Peers}

The concept of integrating edge computing into the mobile network architecture is not new [56. Thereafter, researchers are exploring the idea of using MEC (Mobile Edge Computing) as a gateway for IoT devices to achieve low latency, data aggregation, processing, and real-time application response [57, 58, 59]. The deployment models of MEC range from SCC (Small Cell Cloud) 60, 61, to MMC (Mobile Micro Cloud) 62, MobiScud (Fast Moving Personal Cloud) 63, FMC (Follow Me Cloud) and etc. In all these MEC concepts, the first point of contact between the UE (User Equipment) and the mobile network is SCeNB (Small Cell evolve NodeB) or eNB (evolve NodeB). However, depending upon the MEC architecture the computational and storage resources are located (can be in hardware or virtual form) at SCeNB/eNB for SCC and MCC, and at distributed CN (Core Network) in the case of FMC. However, FMC with decentralized control and distributed architecture is the preferred choice over SCC and MCC [64]. 


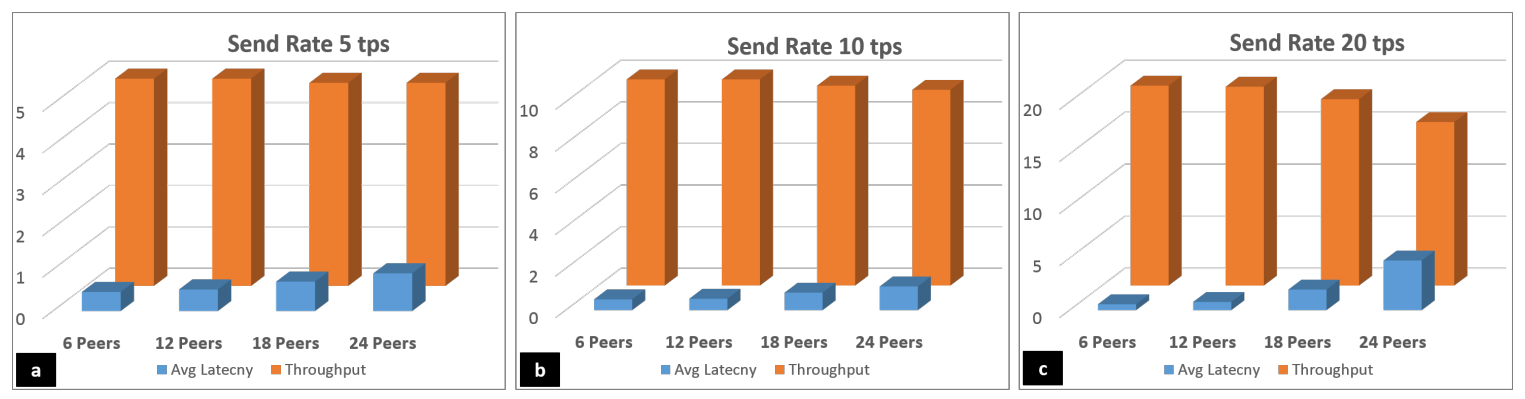

Figure 21: Correlation between number of peers and network throughput at the send rate of (a) 5 tps, (b) 10 tps, and (c) 20 tps

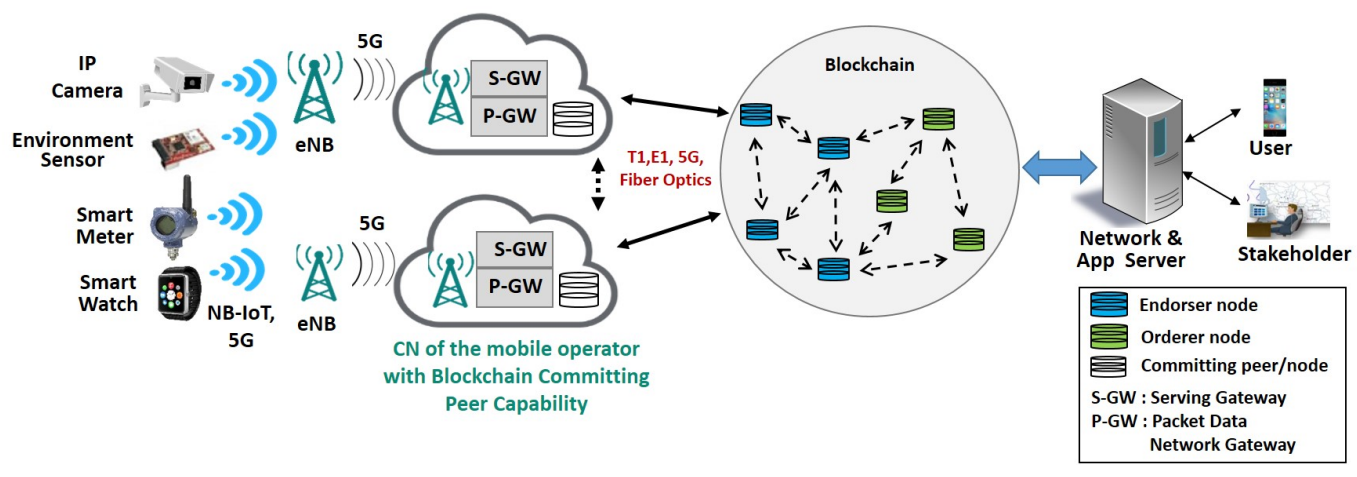

Figure 22: Integration of Blockchain with MEC

We believe that based on the edge computing concept we can integrate blockchain with MEC to relieve end nodes from maintaining many ledgers. In this context, the SCeNB/eNB or CN nodes (in case of FMC architecture) can be harnessed with a suitable blockchain platform to facilitate fast TX settlement and provision of swift data processing and analytics services. Moreover, the end nodes can send queries for data (authorized to them) to the MEC nodes. To realize this concept, we propose a solution based on the FMC model as shown in Figure-22. As of today, almost every inch of a populated area has cellular coverage, and most of the latest IoT devices also support NB-IoT technology. NB-IoT is a sub LTE frequency band, and in the near future, all the telcos (telecommunications companies) will be able to provide NB-IoT services. Moreover, the launch of $5 \mathrm{G}$ mobile network technology is also imminent. Hence, IoT devices can send sensor data to the MEC nodes via NB-IoT/5G. The MEC nodes being resourceful in terms of infrastructure, computational power, storage, and energy can also act as a blockchain committing peer. In this way, we can utilize the existing infrastructure of MEC/cellular networks without incurring excessive costs. The MEC node can then communicate with the endorsing nodes/peers using backhaul network (5G, E1, T1, fiber optics, satellite, etc.) and existing infrastructure at any distance. The inherent communication security of fiber optics, NB-IoT [4, 4G, and 5G 65, 66 technology will also add another layer of security over the blockchain $\mathrm{P}-2-\mathrm{P}$ communication.

Turning a MEC node into a blockchain committing peer will be safe from the data security point of view, as the committing peers do not install and run the SCs. Hence, the SC TX logic will not be visible to them. Moreover, to incentivize the cellular companies for their services, they can be paid some TX fee as a reward in terms of the local digital token, e.g., PrivyCoin. Another advantage of integrating blockchain with MEC model will be ease in mobility management (e.g., handover) of end nodes/user devices if they move 690 throughout the network. 


\subsubsection{Secure IoT Device Integration}

The first element in IoT device security measures is device enrolment, in which only approved devices should be allowed to communicate with the blockchain and call smart contract methods [21. Secondly, all the unnecessary ports on the device should be blocked such as JTAG and UART since any open port can be used by an adversary to access the device and make malicious changes. Finally, most of the commercially available IoT devices such as wireless sensors, do not have a secure execution environment amid low costs. Therefore, the device integrity check should frequently be performed to ensure its legitimacy.

\section{Conclusions and Future Work}

User data generated by today's smart devices ranging from smart watches to smart cars, smart homes, auto-pay systems, ITS, etc., are vulnerable to privacy and security threats. Moreover, users also reserve the right to manage and control access to the data they own. Therefore, in this paper we introduced "PrivySharing", an innovative blockchain-based secure and privacy-preserving data sharing mechanism for smart cities. The proposed strategy ensures that personal/critical user data is kept confidential, securely processed and is exposed to the stakeholders on the need to know basis as per user-defined ACL rules embedded in smart 705 contracts. Moreover, the data owners are rewarded for sharing their data with the stakeholders/third parties. PrivySharing also complies with some of the fundamental EU GDPR requirements, such as data asset sharing, accessibility and purging with data owner's consent. In addition, the experimental results verified that a multi-Ch blockchain solution scales better than a single Ch blockchain system.

Though we have presented all the details of the proposed network architecture and security mechanism, however, as a PoC for this paper, we implemented a part of it. In the future, we aim to extend this work and incorporate the concept of the fog nodes based on existing mobile BTS stations and also devise a mechanism for secure integration of IoT devices with the blockchain network.

\section{Acknowledgements}

We would like to acknowledge Food Agility CRC Ltd for the award of a postgraduate scholarship. Food 715 Agility CRC Ltd is a Cooperative Research Centre funded under the Commonwealth Government CRC program. The CRC Program supports industry-led collaboration between industry, researchers and the community.

\section{References}

[1] D. Lund, C. MacGillivray, V. Turner, M. Morales, Worldwide and Regional Internet of Things (IoT) 2014-2020 Forecast: A Virtuous Circle of Proven Value and Demand, International Data Corporation (IDC), Tech. Rep, 2014.

[2] V. Moustaka, Z. Theodosiou, A. Vakali, A. Kounoudes, Smart Cities at Risk!: Privacy and Security Borderlines from Social Networking in Cities, Athena 357 (2018) 905-910.

[3] K. Zhang, J. Ni, K. Yang, X. Liang, J. Ren, X. S. Shen, Security and Privacy in Smart City Applications: Challenges and Solutions, IEEE Communications Magazine 55 (1) (2017) 122-129.

[4] I. Makhdoom, M. Abolhasan, J. Lipman, R. P. Liu, W. Ni, Anatomy of threats to the internet of things, IEEE Communications Surveys \& Tutorials 21 (2) (2019) 1636-1675.

[5] J. Wurm, K. Hoang, O. Arias, A.-R. Sadeghi, Y. Jin, Security Analysis on Consumer and Industrial IoT Devices, in: Proceedings of the $21^{\text {st }}$ Asia and South Pacific Design Automation Conference (ASP-DAC), IEEE, 2016, pp. 519-524.

[6] A. Bartoli, J. Hernández-Serrano, M. Soriano, M. Dohler, A. Kountouris, D. Barthel, Security and Privacy in your Smart City, in: Proceedings of the Barcelona Smart Cities Congress, Vol. 292, 2011, pp. 1-6.

[7] L. Cui, G. Xie, Y. Qu, L. Gao, Y. Yang, Security and Privacy in Smart Cities: Challenges and Opportunities, IEEE Access 6 (2018) 46134-46145. 
[8] D. Puthal, S. Nepal, R. Ranjan, J. Chen, Threats to Networking Cloud and Edge Datacenters in the Internet of Things, IEEE Cloud Computing 3 (3) (2016) 64-71.

[9] S. Sara, N. Michael, 2018. Facebook has been worried about data leaks like this since it went public in 2012, (Last accessed 11 September 2018). URL https://www.cnbc.com/2018/04/12/facebook-warned-of-data-breaches-years-ago-when-it-went-public-in2012.html

[10] S. Jason, 2019. Hundreds of millions of Facebook user records were exposed on Amazon cloud server, (Last accessed 19 April 2019). URL https://www.cbsnews.com/news/millions-facebook-user-records-exposed-amazon-cloud-server/

[11] S. Sara, 2018. A Google bug exposed the information of up to 500,000 users, (Last accessed 30 Dec 2018). URL https://www.cnbc.com/2018/10/08/google-bug-exposed-the-information-of-up-to-500000-users.html

[12] GDPR, 2018. General Data Protection Regulation, (Last accessed 3 Jan 2019). URL https://gdpr-info.eu/

[13] R. A. Michelin, A. Dorri, M. Steger, R. C. Lunardi, S. S. Kanhere, R. Jurdak, A. F. Zorzo, SpeedyChain: A Framework for Decoupling Data from Blockchain for Smart Cities, in: Proceedings of the $15^{\text {th }}$ EAI International Conference on Mobile and Ubiquitous Systems: Computing, Networking and Services, ACM, 2018, pp. 145-154.

[14] P. K. Sharma, J. H. Park, Blockchain Based Hybrid Network Architecture for the Smart City, Future Generation Computer Systems 86 (2018) 650-655.

[15] M. A. Rahman, M. M. Rashid, M. S. Hossain, E. Hassanain, M. F. Alhamid, M. Guizani, Blockchain and IoT-based Cognitive Edge Framework for Sharing Economy Services in a Smart City, IEEE Access 7 (2019) 18611-18621.

[16] K. Biswas, V. Muthukkumarasamy, Securing Smart Cities using Blockchain Technology, in: Proceedings of the $14^{\text {th }}$ International Conference on Smart City High Performance Computing and Communications, IEEE, 2016, pp. 1392-1393.

[17] D. A. Kountché, J.-M. Bonnin, H. Labiod, The Problem of Privacy in Cooperative Intelligent Transportation Systems (C-ITS), in: Proceedings of the Computer Communications Workshops (INFOCOM WKSHPS), IEEE, 2017, pp. 482-486.

[18] F. Haidar, A. Kaiser, B. Lonc, On the Performance Evaluation of Vehicular PKI Protocol for V2X Communications Security, in: Proceedings of the $86^{t h}$ Vehicular Technology Conference (VTC-Fall), IEEE, 2017, pp. 1-5.

[19] A. Dorri, S. S. Kanhere, R. Jurdak, Blockchain in Internet of Things: Challenges and Solutions, arXiv preprint arXiv:1608.05187.

[20] A. Dorri, S. Kanhere, R. Jurdak, P. Gauravaram, Blockchain for IoT Security and Privacy: The Case Study of a Smart Home, in: Proceedings of the $2^{\text {nd }}$ Workshop on Security, Privacy, and Trust in the Internet of Things (PERCOM), IEEE, 2017, pp. 1-6.

[21] I. Makhdoom, M. Abolhasan, H. Abbas, W. Ni, Blockchain's Adoption in IoT: The Challenges, and a Way Forward, Journal of Network and Computer Applications 125 (2018) $251-279$.

[22] V. Buterin, et al., A Next-generation Smart Contract and Decentralized Application Platform, Whitepaper.

[23] S. Huh, S. Cho, S. Kim, Managing IoT Devices using Blockchain Platform, in: Proceedings of the $19^{\text {th }}$ International Conference on Advanced Communication Technology (ICACT), IEEE, 2017, pp. 464-467.

[24] B. Leiding, P. Memarmoshrefi, D. Hogrefe, Self-managed and Blockchain-based Vehicular Ad-hoc Networks, in: Proceedings of the International Joint Conference on Pervasive and Ubiquitous Computing: Adjunct, ACM, 2016, pp. 137-140.

[25] Y. Zhang, J. Wen, The IoT Electronic Business Model: Using Blockchain Technology for the Internet of Things, Peer-to-Peer Networking and Applications (2016) 1-12. 
[43] A. Dorri, S. S. Kanhere, R. Jurdak, Mof-bc: A memory optimized and flexible blockchain for large scale networks, Future Generation Computer Systems 92 (2019) 357-373. [44] C. Roulin, A. Dorri, R. Jurdak, S. Kanhere, On the activity privacy of blockchain for iot, arXiv preprint arXiv:1812.08970 (2018) 1-8.

26] K. N. Krishnan, R. Jenu, T. Joseph, M. Silpa, Blockchain Based Security Framework for IoT Implementations, in: Proceedings of the International CET Conference on Control, Communication, and Computing (IC4), IEEE, 2018, pp. 425-429.

[27] Y. Qian, Z. Liu, J. Yang, Q. Wang, A Method of Exchanging Data in Smart City by Blockchain, in: Proceedings of the $16^{\text {th }}$ International Conference on Smart City, IEEE, 2018, pp. 1344-1349.

[28] N. B. Truong, K. Sun, G. M. Lee, Y. Guo, GDPR-Compliant Personal Data Management: A Blockchain-based Solution, IEEE Transactions on Inromation Forensics and Security (2019) 1-13.

[29] B. Faber, G. C. Michelet, N. Weidmann, R. R. Mukkamala, R. Vatrapu, BPDIMS: A blockchain-based personal data and identity management system, in: Proceedings of the $52^{\text {nd }}$ Hawaii International Conference on System Sciences (HICSS), IEEE, 2019, pp. 6855-6864.

[30] R. Neisse, G. Steri, I. Nai-Fovino, A blockchain-based approach for data accountability and provenance tracking, in: Proceedings of the $12^{t h}$ International Conference on Availability, Reliability and Security, ACM, 2017, pp. 1-10.

[31] K. Rantos, G. Drosatos, K. Demertzis, C. Ilioudis, A. Papanikolaou, A. Kritsas, ADvoCATE: A Consent Management Platform for Personal Data Processing in the IoT Using Blockchain Technology, in: Proceedings of the International Conference on Security for Information Technology and Communications, Springer, 2018, pp. 300-313.

[32] N. Kaaniche, M. Laurent, A blockchain-based data usage auditing architecture with enhanced privacy and availability, in: Proceedings of the $16^{t h}$ International Symposium on Network Computing and Applications (NCA), IEEE, 2017, pp. 1-5.

[33] W. J. Gordon, C. Catalini, Blockchain technology for healthcare: facilitating the transition to patient-driven interoperability, Computational and structural biotechnology journal 16 (2018) 224-230.

[34] Hyperledger-Fabric, 2019. Smart Contracts and Chaincode, (Last accessed 4 Apr 2019). URL https://hyperledger-fabric.readthedocs.io/en/release-1.4/smartcontract/smartcontract.html

[35] Hyperledger-Fabric, 2019. Blockchain Network, (Last accessed 4 Apr 2019). URL https://hyperledger-fabric.readthedocs.io/en/release-1.4/network/network.html

[36] O. Mazhelis, A. Hämäläinen, T. Asp, P. Tyrväinen, Towards Enabling Privacy Preserving Smart City Apps, in: Proceedings of the International Smart Cities Conference (ISC2), IEEE, 2016, pp. 1-7.

[37] I. Makhdoom, M. Abolhasan, W. Ni, Blockchain for IoT: The Challenges and a Way Forward, in: Proceedings of the $15^{\text {th }}$ International Joint Conference on e-Business and Telecommunications - Volume 2: SECRYPT, INSTICC, SciTePress, 2018, pp. 428-439.

[38] J. C. Anderson, J. Lehnardt, N. Slater, CouchDB: The Definitive Guide: Time to Relax, O'Reilly Media, Inc., 2010 .

[39] A. Dent, Getting started with LevelDB, Packt Publishing Ltd, 2013.

[40] M. Marchesi, L. Marchesi, R. Tonelli, An agile software engineering method to design blockchain applications, in: Proceedings of the $14^{\text {th }}$ Central and Eastern European Software Engineering Conference Russia, ACM, 2018 , pp. 1-8.

[41] N. Apthorpe, D. Reisman, N. Feamster, A smart home is no castle: Privacy vulnerabilities of encrypted iot traffic, arXiv preprint arXiv:1705.06805 (2017) 1-6.

[42] Hyperledger-Fabric, 2019. Identity, (Last accessed 9 Oct 2019). URL https://hyperledger-fabric.readthedocs.io/en/release-1.4/identity/identity.html 
[48] Hypothesis Testing (P-Value Approach) (Last accessed 01 Sep 2019).

1. URL https://onlinecourses.science.psu.edu/statprogram/reviews/statistical-concepts/hypothesis-testing/pvalue-approach

[49] C. Ferris, 2019. Hyperledger Fabric performance and scale (Last accessed 01 Sep 2019). URL https://www.ibm.com/blogs/blockchain/2019/01/answering-your-questions-on-hyperledger-fabricperformance-and-scale/

[50] E. Androulaki, A. Barger, V. Bortnikov, C. Cachin, K. Christidis, A. De Caro, D. Enyeart, C. Ferris, G. Laventman, Y. Manevich, et al., Hyperledger fabric: a distributed operating system for permissioned blockchains, in: Proceedings of the $13^{\text {th }}$ EuroSys Conference, ACM, 2018, pp. 1-15.

[51] S. A. Kumar, T. Vealey, H. Srivastava, Security in internet of things: Challenges, solutions and future directions, in: Proceedings of the $49^{\text {th }}$ Hawaii International Conference on System Sciences (HICSS), IEEE, 2016, pp. 5772-5781.

[52] A.-R. Sadeghi, C. Wachsmann, M. Waidner, Security and privacy challenges in industrial Internet of Things, in: Proceedings of the $52^{\text {nd }}$ ACM/EDAC/IEEE Design Automation Conference (DAC), 2015, pp. 1-6.

[53] J. E. Dunn, 2016. Krebs DDoS aftermath: industry in shock at size, depth and complexity of attack (Last accessed 25 Aug 2019).

URL https://www.computerworld.com/article/3427227/krebs-ddos-aftermath--industry-in-shock-at-size-depth-and-complexity-of-attack.html

[54] M. Rostami, F. Koushanfar, R. Karri, A primer on hardware security: Models, methods, and metrics, Proceedings of the IEEE 102 (8) (2014) 1283-1295.

[55] G. Hernandez, O. Arias, D. Buentello, Y. Jin, Smart nest thermostat: A smart spy in your home, Black Hat USA (2014).

[56] Y. C. Hu, M. Patel, D. Sabella, N. Sprecher, V. Young, Mobile edge computingA key technology towards 5G, ETSI white paper 11 (11) (2015) 1-16.

[57] O. Salman, I. Elhajj, A. Kayssi, A. Chehab, Edge computing enabling the Internet of Things, in: Proceedings of the $2^{\text {nd }}$ World Forum on Internet of Things (WF-IoT), IEEE, 2015, pp. 603-608.

[58] S. Abdelwahab, B. Hamdaoui, M. Guizani, T. Znati, Replisom: Disciplined tiny memory replication for massive IoT devices in LTE edge cloud, Internet of Things Journal 3 (3) (2016) 327-338.

[59] X. Sun, N. Ansari, EdgeIoT: Mobile edge computing for the Internet of Things, IEEE Communications Magazine 54 (12) (2016) 22-29.

[60] FP7 European Project, 2012., Distributed Computing, Storage and Radio Resource Allocation Over Cooperative Femtocells (TROPIC), (Last accessed 22 Apr 2019).

URL http://www.ict-tropic.eu/

[61] F. Lobillo, Z. Becvar, M. A. Puente, P. Mach, F. L. Presti, F. Gambetti, M. Goldhamer, J. Vidal, A. K. Widiawan, E. Calvanesse, An architecture for mobile computation offloading on cloud-enabled LTE small cells, in: Proceedings of the Wireless Communications and Networking Conference Workshops (WCNCW), IEEE, 2014, pp. 1-6.

[62] S. Wang, G.-H. Tu, R. Ganti, T. He, K. Leung, H. Tripp, K. Warr, M. Zafer, Mobile micro-cloud: Application classification, mapping, and deployment,, in: Proceedings of the Annual Fall Meeting of ITA (AMITA), 2013, pp. $1-7$. 
[63] K. Wang, M. Shen, J. Cho, A. Banerjee, J. Van der Merwe, K. Webb, Mobiscud: A fast moving personal cloud in the mobile network, in: Proceedings of the $5^{\text {th }}$ Workshop on All Things Cellular: Operations, Applications and Challenges, ACM, 2015, pp. 19-24.

[64] P. Mach, Z. Becvar, Mobile edge computing: A survey on architecture and computation offloading, IEEE Communications Surveys \& Tutorials 19 (3) (2017) 1628-1656.

[65] I. Ahmad, T. Kumar, M. Liyanage, J. Okwuibe, M. Ylianttila, A. Gurtov, Overview of 5G security challenges and solutions, Communications Standards Magazine 2 (1) (2018) 36-43.

[66] M. A. Ferrag, L. Maglaras, A. Argyriou, D. Kosmanos, H. Janicke, Security for 4G and 5G cellular networks: A survey of existing authentication and privacy-preserving schemes, Journal of Network and Computer Applications 101 (2018) 55-82. 